\title{
Chemical Modification of Polymer Surfaces for Advanced Triboelectric Nanogenerator Development
}

\author{
Yanhao $\mathrm{Yu}^{1}$, Xudong Wang ${ }^{1,2, *}$
}

1. Department of Materials Science and Engineering, University of Wisconsin-Madison,

$$
\text { Madison, WI 53706, USA }
$$

2. Beijing Institute of Nanoenergy and Nanosystems, Chinese Academy of Sciences; National Center for Nanoscience and Technology (NCNST), Beijing, 100083, P. R. China

\section{Email: xudong.wang@wisc.edu}

\begin{abstract}
Triboelectric nanogenerator (TENG) is a newly developed technique for harvesting mechanical energy from ambient environment with sparkly high output and extremely flexible structural designs. The operation of TENGs is based on the combined effects of triboelectrification and electrostatic induction. The charge density on triboelectric surfaces (mostly polymers) sets the foundation of TENG output. Meanwhile, the charge density on polymer surface is closely related to the surface chemical property. Therefore, engineering the surface chemical environment by appropriate functionalization is the most fundamental approach in controlling the TENG outputs. This article systematically reviews recent processes of chemical modifications of triboelectric polymers for advanced TENG developments. According to different functionalization techniques, four categories of chemical modifications, including fluorinated surface, ion injection, sequential infiltration synthesis and molecular-targeting
\end{abstract}


functionalization are thoroughly reviewed, and their contributions to TENG performance are discussed.

Keywords: Triboelectric Nanogenerator; Surface Modification; Polymer Surfaces; Mechanical Energy Harvesting 


\section{Introduction}

The rapid development of mobile electronics has reconstructed the landscape of energy consumption. Miniaturization of energy sources with high portability and sustainability is greatly desired for powering a large variety of personal electronics and implantable biomedical devices. Currently, batteries are the predominant choice of such power sources. However, they may not be the ultimate solution since their operational lifetimes are limited and regular recharging or replacing is necessary, which give rises to their incompatibility, incompetency and even environmental concerns.[1] Based on this regard, self-powered charging system would be a desired power source for modern electronics. Ambient environmental mechanical energy is considered abundant and the most accessible energy source for powering small electronics.[2] Among many endeavors of harvesting mechanical energy effectively, the nanogenerator was invented using piezoelectric nanostructures as the functional building blocks, which offers high sensitivity, long life time, and potential superior efficiency.[3] During the last decade, numerous piezoelectric nanogenerators (PENGs) were developed to harvest energy from physical deflection,[4,5] acoustic waves,[6,7] air and fluid flow,[8-10] and body movements (e.g. walking, talking and breathing).[11-13] In 2012, triboelectric nanogenerator (TENG) was first reported on the basis of the traditional electrostatic energy harvesting principles.[14] Owing to its simple design and scalable/integratable nature, the area and volume power density of TENGs rapid reached $500 \mathrm{~W} / \mathrm{m}^{2}$ and $15 \mathrm{MW} / \mathrm{m}^{3}$, respectively within a few years.[15] An instantaneous conversion efficiency of $70.6 \%$ and a total conversion efficiency of $85 \%$ were achieved.[16,17] The high power output and large energy conversion efficiency made TENGs feasible in driving various commercial electronics, such as cell phones,[18,19] light bulbs,[15,19] alarms,[20,21] pacemakers,[22] and a large number of sensors[23-28] and light emitting diodes (LEDs),[29- 
34]According to the device configuration, TENGs can be categorized into four modes: vertical contact-separation mode, contact-sliding mode, single-electrode mode, and freestanding triboelectric-layer mode,[35] which cover the functions of TENGs in converting various energy forms including water waves,[36-38] wind,[39,40] rain droplets,[30,41] vehicle[32,42] and human movement.[17,43,44] These two advantages promise TENGs in not only developing practical self-powered energy systems but also generating electricity at large scale. The development and broad applications of TENGs were extensively summarized in a number of recent reviews.[1,2,35,45-48]

The operation of TENG is on the basis of two combined effect of triboelectrification and electrostatic induction. The triboelectrification is the phenomenon that a material becomes electrically charged after intimate contact with a different object. When two dissimilar surfaces with distinct triboelectric polarity (i.e. tendency to gain or lose electrons) touch each other, electrons, holes or other charged ions/molecules transfer across the interface to compensate the difference of surface potentials. This charge separation is the primary driving force of mechanical to electric energy conversion in TENGs. Triboelectric materials (mostly polymer) are usually attached to metal electrodes, where electrostatic charges are induced. Once the device capacitance changes by displacement, current flow occurs between the conductive electrodes and thus outputs electric power. Therefore, controlling the charge density on the triboelectric surfaces is the most fundament strategy for improving the performance of TENG. In general, the charge density is determined by the amount of charge carrying locations and the tendency of these sites to gain or lose electron. These two factors can be regulated by morphologically and chemically modifying the triboelectric polymers. The number of charge carriers can be simply increased by enlarging the surface roughness factor. For instance, morphologies like nanowires,[49- 
51]nanoparticles[31,52] or other nanoscale patterns[53-59] are frequently adopted to raise the surface area of triboelectric materials and thus enable more charge carrying site. On the other hand, the tendency of these sites can be controlled by altering their chemical properties. The objective of this article is to give a review of most recent advances in chemically engineering polymer surface and to discuss the contribution of four different modification techniques to enhance TENG performances.

\section{Fluorinated Surfaces}

The triboelectrification process exists in various material pairs. To maximize the charge transfer density, efficient TENGs are built on two surfaces with the most distinguished ability to gain or refuse electrons. Different surfaces' tendency of receiving and losing electrons were listed in several triboelectric series,[1,29,60] which are the guidance of material selection in TENG designing. Fundamentally, this ability/tendency is closely related to the electron affinity of the surface atoms. For instance, most electron-attracting polymers contain elements with strong electron affinity, such as fluorine (F). Therefore, one intuitively expected approach to increase the charge density is introducing extra $\mathrm{F}$ atoms onto the polymer surface by chemical modifications, so called fluorinated surfaces. In principle, surfacefluorination is achieved by chemically grafting F-containing unit, such as $\mathrm{CF}_{3}$ group through either solution reactionsor vapor treatments.

Shin et al. manipulated the triboelectric polarities of polyethylene terephthalate (PET) film by functionalizing its surface with positively charged amino group $\left(-\mathrm{NH}_{3}\right)$ and negatively charged $\mathrm{CF}_{3}$ group using poly-L-lysine solution and trichloro $(1 \mathrm{H}, 1 \mathrm{H}, 2 \mathrm{H}, 2 \mathrm{H}-$ perfluorooctyl)silane (FOTS) vapor, respectively.[61] The modification process started from the cleaning of PET substrates. 
After that, the PET substrates were treated in oxygen plasma for $100 \mathrm{~s}$, resulting in the formation of reactive hydroxyl groups (-OH) on the surface. At last, two pieces of plasma-treated PETs were individually soaked in poly-L-lysine solution for 5 min and exposed in FOTs vapor environment at $95^{\circ} \mathrm{C}$ for $1 \mathrm{~h}$. During this process, target molecules (i.e. poly-L-lysine and FOTs) were anchored on the PET surface by covalently bonding with the reactive hydroxyl groups. To verify the surface functionalization, water contact angles of the pristine and treated samples were analyzed through static sessile drop experiments. The contact angles of poly-L-lysine-treated PET (P-PET), pristine PET, and FOTs-treated PET (F-PET) were $44^{\circ}, 81^{\circ}$ and $123^{\circ}$, indicating the hydrophilic and hydrophobic properties, respectively. The hydrophilic characteristic of PPET was attributed to the positively charged amino groups that actively interact with the polar hydrogen bonds of water molecules. The hydrophobic feature of F-PET was a consequence of the $-\mathrm{CF}_{3}$ group in the graphed FOTs molecules, which has low surface energy to interact water molecules. These results confirmed the presence of $-\mathrm{NH}_{3}$ group on the P-PET and $-\mathrm{CF}_{3}$ group on the F-PET surface.

After the chemical modifications, P-PET and F-PET demonstrated higher tendency to lose and gain electron due to the $-\mathrm{NH}_{3}$ group and the fluorinated surface, respectively. In principle, TENG with these two surfaces should deliver significant electric output considering the enlarged difference of triboelectric polarities. Figures 1a and b compare the voltage output of TENGs with different friction pairs: (i) PET:PET, (ii) PET:P-PET, (iii) PET:F-PET, and (iv) P-PET:F-PET. A smallest $V_{o c}$ of $\sim 4 \mathrm{~V}$ was produced in pair (i) with a device area of $4 \mathrm{~cm}^{2}$ and under an applied force of $0.5 \mathrm{MPa}$. P-PET and F-PET could improve the $V_{\text {oc }}$ to $\sim 110 \mathrm{~V}$ and $\sim 205 \mathrm{~V}$, respectively. The maximum $V_{o c}$ of $\sim 330 \mathrm{~V}$ was obtained with the TENG assembled from pair (iv). That was $\sim 80$ times enlargement compared to the $V_{o c}$ of PET:PET pair, implying the effectiveness of this 
amino and fluorine functionalization methods. The modified PET surfaces exhibited superior stability owing to the tight chemical bonds. No reduction of voltage or current output was observed during the periodical measurement of TENG with pair (iv) for over 4 weeks (Figure 1c). A $V_{o c}$ of $\sim 300 \mathrm{~V}$ and a short-circuit current density $\left(J_{s c}\right)$ of $\sim 200 \mathrm{~mA} / \mathrm{m}^{2}$ can be maintained within the entire time interval. These electric outputs were able to lighting up 250 blue LEDs with a rectified bridge circuit to convert alternating current (AC) to direct current (DC).

Similar FOTs functionalization was applied to polypropylene (PP) nanowires (NWs). Feng et al. fabricated PP NW array with anodic aluminum oxide (AAO) template and chemically modified the NW surfaces with two fluorine-containing molecules including FOTs and poly(2,2,3,4,4,4-hexafluorobutyl-5-((3,4-dihydroxyphenethyl)amino)-2,2,4,4-tetramethyl-5oxopentanoate)fluoropolymer (Pdop-F). [62] Compared with the Pdop-F, FOTs was found to be more effective in implementing fluorine onto PP NW surfaces since the fluorine content in FOTs (48.6 wt\%) was much higher than Pdop-F (23.8 wt\%). Consequently, the charge density of FOTs treated PP NWs $\left(65 \mu \mathrm{C} / \mathrm{m}^{2}\right)$ was about two times larger than that of Pdop-F treated PP NWs (30 $\mu \mathrm{C} / \mathrm{m}^{2}$ ). Both of these two values are substantially higher than the pristine PP NWs ( $3.3 \mu \mathrm{C} / \mathrm{m}^{2}$ ). As a result, FOTs treated PP NWs achieved the highest voltage output of $1900 \mathrm{~V}$ and current density output of $19 \mathrm{~mA} / \mathrm{cm}^{2}$, which were sufficient to powering 372 red LEDs.

Another popular way to introduce fluorine is fluorocarbon plasma, i.e., employing fluorocarbon (e.g. $\mathrm{CF}_{4}$ and $\mathrm{C}_{4} \mathrm{~F}_{8}$ ) plasma gas to treat the polymer surfaces. During this process, fluorinated and roughened surface can be achieved simultaneously. Zhang et al. reported a onestep fluorocarbon plasma treatment for chemically modifying polydimethylsiloxane (PDMS) film.[63] An inductively coupled plasma (ICP) machine was employed to perform the plasma treatment with $\mathrm{C}_{4} \mathrm{~F}_{8}$ as the active gas and fluorine source. Figures 2a-c are the schematic and 
scanning electron microscope (SEM) pictures of the hierarchical PDMS film used in the plasma treatment and TENG fabrications. After the fluorocarbon plasma, small holes emerged on the surfaces of PDMS islands. Figure 2d shows the voltage outputs of TENGs that assembled from pristine PDMS and PDMS with different cycles of $\mathrm{C}_{4} \mathrm{~F}_{8}$ plasma treatment (12 s per cycles). 1cycle treatment sharply increased the voltage from $124 \mathrm{~V}$ to $193 \mathrm{~V}$. Subsequently, the voltage output could be further improved with the increase of treating cycles, which was ascribed to the increasing amount of $\mathrm{F}$ atoms. A maximum voltage of $265 \mathrm{~V}$ was attained with 8-cycle treatment. Further extending the treating cycles resulted in a decrease of voltage output. This output reduction was attributed to two reasons: the degradation of electrostatic induction caused by the thicker fluorocarbon layer; and the influence of the roughness factor on PDMS surfaces. From the SEM observation (Figure 2c), 1-cycle treatment can induce significant etching on the PDMS surfaces. This roughness was assumed to contribute to the output improvement. Within 8 cycles, the PDMS surface was found to be partially covered by fluorocarbon and the etching remained effective. After 8 cycles, fluorocarbon polymeric layer capped the entire PDMS surface and thus reduced the roughness factor, diminishing the etching contribution.

Since the electric output of this TENG was largely improved, the electron binding energy of PDMS was expected to be appreciably enhanced by the $\mathrm{C}_{4} \mathrm{~F}_{8}$ treatment. This assumption was verified by the first principle calculations. The relative ability of losing one electron of pristine PDMS and $\mathrm{C}_{4} \mathrm{~F}_{8}$ modified PDMS were analyzed by calculating the vertical ionization energies of two model complexes $\left(\mathrm{CH}_{3}\right)_{3} \mathrm{Si}\left(\mathrm{OSi}\left(\mathrm{CH}_{3}\right)_{2}\right)_{2} \mathrm{OSi}\left(\mathrm{CH}_{3}\right)_{3}$ and $\mathrm{CF}_{3}\left(\mathrm{CF}_{2}-\mathrm{CF}_{2}\right)_{2} \mathrm{CF}_{3}$, respectively. As shown in Figure 2e and $\mathrm{f}$, the ionization energies of PDMS and $\mathrm{C}_{4} \mathrm{~F}_{8}$ modified PDMS were 8.98 and $12.31 \mathrm{eV}$, respectively, affirming the latter has larger electron binding energy and higher tendency to gain electrons. 
If the PDMS film is thin and treated before curing, this fluorocarbon plasma approach could create wrinkled PDMS film with chemically modified surfaces.[64] Figure 3a depicted the fabrication procedure of a wrinkled PDMS film and the corresponding TENG setup. PET/ITO film was used as the substrate for the spin-coated PDMS film as well as the counter electrode of the TENG. SEM and laser scanning microscope (LSM) characterizations in Figure 3b demonstrated the curved topology of PDMS with an average width of $\sim 15 \mu \mathrm{m}$ and height of $\sim 12$ $\mu \mathrm{m}$. The formation of this wrinkled surface was probably caused by the velocity from the fluorocarbon polymer. The disordered momentum of fluorocarbon ions would introduce the random deformation of PDMS. Another possible reason could be the different surface tension between the PDMS and the fluorocarbon polymer layer during the curing process.

The chemical composition and bonding state of the modified PDMS film was explored by Xray photoelectron spectroscopy (XPS). Strong fluorine peak was identified in the spectrum of $\mathrm{C}_{4} \mathrm{~F}_{8}$ treated PDMS, evidencing the appearance of fluorine atom on the surface. The element contents that were detected by XPS varied with the plasma treating time. When the plasma treating time increased from $50 \mathrm{~s}$ to $150 \mathrm{~s}$, O content decreased from $13.29 \%$ to $4.4 \%$, and $\mathrm{F}$ increased from $25.06 \%$ to $42.37 \%$. Combined with the wrinkled surface, these extra fluorine atoms could greatly enhance the electric output of TENG. Figures 3c and d manifest the voltage, current and charge outputs of the flat PDMS, $\mathrm{C}_{4} \mathrm{~F}_{8}$-treated flat PDMS and $\mathrm{C}_{4} \mathrm{~F}_{8}$-treated wrinkled PDMS. Compared with the untreated PDMS, $\mathrm{C}_{4} \mathrm{~F}_{8}$ flat PDMS and wrinkled PDMS improved the voltage output from 120 to 150 and $240 \mathrm{~V}$, corresponding to $25 \%$ and $100 \%$ enhancement, respectively. The peak current and charge outputs of the $\mathrm{C}_{4} \mathrm{~F}_{8}$-treated wrinkled PDMS in a single cycle were $182 \mu \mathrm{A}$, and $528 \mathrm{nC}$, respectively, which were able to lighting up 72 commercial blue LEDs. 
In addition to PDMS, PET surface can also be chemically modified by the fluorocarbon plasma approach.[65] In this case, a mixture of carbon tetrafluoride $\left(\mathrm{CF}_{4}\right)$ and oxygen $\left(\mathrm{O}_{2}\right)$ gases were used as the plasma source. Analogous to $\mathrm{C}_{4} \mathrm{~F}_{8}$ plasma, $\mathrm{CF}_{4}$ treatment could yield both chemical modification by fluorination and physical modification by roughening the polymer surface in nanoscale (Figure 4a). The electric potential of the PET before and after modification were measured with Kelvin probe force microscopy (KPFM). The surface potential was increased from $41 \mathrm{mV}$ to $105 \mathrm{mV}$ after the plasma treatment. This improvement was attributed to the enlarged surface charge density, which was induced by the fluorine modification and increased surface area. According to topography characterizations from the atomic force microscopy (AFM), the unmodified PEF surface was very smooth with an average roughness of $2.2 \mathrm{~nm}$ and a root mean square roughness of $2.8 \mathrm{~nm}$. After the plasma treatment, the average and root mean square roughness were increased to 27.4 and $34.4 \mathrm{~nm}$, respectively. The maximum profile peak height was as high as $320 \mathrm{~nm}$. This surface roughening effect was a result of the anisotropic etching by ionic bombardment due to the applied bias.[66] Based on the fundamental mechanism of TENG, considerable electric output could be anticipated after establishing the rough and fluorinated surfaces. Figures $4 \mathrm{~b}-\mathrm{d}$ present the $V_{o c}, I_{s c}$ and charge transfer amount $(Q)$ of pristine PET, plasma treated PET and polytetrafluoroethylene (PTFE) film. The $V_{o c}, I_{s c}$ and $Q$ of the modified PET reached approximately $220 \mathrm{~V}, 45 \mu \mathrm{A}$ and $130 \mathrm{nC}$, respectively, which were over two times larger than those of the pristine PET film. These electric outputs even outperformed the PTFE film, which is considered as the most efficient triboelectric polymer with the strongest tendency to gain electrons,[1] reinforcing the effectiveness of the $\mathrm{CF}_{4}$ plasma modification approach. 
The fundamental mechanism of the $\mathrm{CF}_{4}$ modification was studied in this work. XPS was employed to detect the elemental compositions and their local chemical environments on the pristine and modified PET surfaces. Strong fluorine peak emerged in the full survey spectrum of the modified PET sample and no fluorine peak was found from the pristine PET film. C 1s peaks were individually scanned with high resolution. As shown in Figure 4e, for the pristine PET, $66.3 \%$ of $\mathrm{C}-\mathrm{C}, 18.4 \%$ of $\mathrm{C}-\mathrm{O}$ and $15.3 \%$ of $\mathrm{C}=\mathrm{O}$ bond can be recognized in the deconvoluted curves, agreeing well with the PET molecular formula. On the other hand, the deconvolution of C 1s peak in the modified PET exhibited apparently reduced amount of C-C (23.3\%), increased percentage of $\mathrm{C}-\mathrm{O}$ (33.9\%) and similar amount of $\mathrm{C}=\mathrm{O}$ (13.3\%) (Figure 4f). Three new peaks were identified including $\mathrm{CF}$ (9.5\%), $\mathrm{CF}_{2}$ (11.4\%) and $\mathrm{CF}_{3}$ (8.6\%). Based on these measurements, two possible reaction mechanisms were proposed for this fluorination process. During the plasma discharging, $\mathrm{CF}_{4}$ gas was decomposed into free radicals such as ${ }^{-} \mathrm{CF}_{3} \mathrm{and}: \mathrm{CF}_{2}$. These active radicals were eager to recombine into $\mathrm{CF}_{4}$. However, the presence of $\mathrm{O}_{2}$ suppressed this process by forming $\cdot \mathrm{COF}$ and $\mathrm{CO}_{2}$, and releasing more $\cdot \mathrm{F}$ free radicals.[67] These free radicals reacted with PET macromolecules via two possible routes, named as "implantation" and "graphing" (Figure 4g). For the "implantation" path, free radicals replaced hydrogen or added to pi bonds of the aromatic rings, forming reactive saturated intermediates.[68] For the "graphing" path, : $\mathrm{CF}_{2}$ radicals first replaced hydrogen, then further reacted with other free $: \mathrm{CF}_{2}$, and eventually bonded to $-\mathrm{CF}_{2}-\mathrm{CF}_{2}$-chains.[69]

In summary, developing fluorinated surfaces is a straightforward and effective approach to chemically modifying triboelectric polymers. For the fluorocarbon plasma process, chemically and morphologically modified surfaces can be achieved simultaneously. Since the electron affinity of fluorine atom is extraordinarily high, fluorination could significantly enhance the 
materials' ability of holding electrons, giving rise to drastic performance gain of TENG devices. However, for the same reason, this method is only effective for improving polymers with negative triboelectric polarity. Although the majority of current TENGs are built on polymers with negative polarity, materials with positive polarity are frequently used for certain applications (e.g. cottons for wearable electronics). Fluorination would obviously jeopardize their performance by suppressing their tendency of losing electrons.

\section{Ion Injection}

Ion injection is another chemical modification approach with relatively broad source and target selections since a number of ions could be the charge source. Typically, the triboelectric charge density is not able to reach the level of maximum surface charge density (MSCD), which is determined by the breakdown electric field of air, due to the limited potential differences between two conventional friction surfaces. This means most polymer surfaces can handle extra charges besides the portion acquired from triboelectrification. One intuitive sense to introducing additional charges is directly injecting charged ions into desired surfaces. Through electrostatic absorption, the injected charged ions can remain in the dielectric polymers for years. [70] The most common method to conduct ion injection is plasma treatment. Reactive ion etching (RIE) is a widely used technique to increase the surface roughness of polymer by generating NW morphology with argon and oxygen as the plasma gas.[71] During this process, corresponding argon and oxygen ions are spontaneously injected into the triboelectric polymer, resulting in chemical modulation of surface states. From this point of view, all polymer NWs that made from RIE will experience chemical modification by ion injection. 
In addition to the plasma treatment, Wang et al. employed ionized-air gun to controllably implanting extra charges to the fluorinated ethylene propylene (FEP) surface and systematically studied the MSCD of FEP polymer.[70] Compared to other charge implantation methods such as ICP and corona discharging,[72] this hand-sized ionized-air gun is simple and convenient. Ionized-air gun can produce ions with both polarities via triggering the discharge of air inside the gun. The polarity of ion can be manipulated by either squeezing or releasing the trigger. Negative charges $\left(\mathrm{CO}^{3-}, \mathrm{NO}^{3-}, \mathrm{NO}^{2-}, \mathrm{O}^{3-}\right.$ and $\left.\mathrm{O}^{2-}\right)$ were selected in this work since FEP has strong tendency to gain electrons (Figure 5a). In order to achieve the MSCD for highperformance TENG, the metal electrode that attached to the back of polymer layer was grounded. Under this circumstance, the injected negative charges are able to drive equal amount of electron away from the metal electrode to ground, leaving positive charges in the electrode. As a consequence, the electrical field of the double layer charge will be confined only within the FEP film. Since the dielectric strength of FEP is two orders of magnitudes larger than that of air, the grounded connection could allow a much larger charge density compared to the electrode-free FEP film, in which the maximum charge density will be limited by the breakdown voltage of the air.

To investigate the MSCD of FEP film, the charge density on the charge-injected polymer surface was instantaneously monitored by a Coulomb meter, which could measure the charge flow between the bottom electrode and ground (inset of Figure 5b). As shown in Figure 5b, every period of ion injection induced a charge transferring process with an area density of $\sim 40 \mu \mathrm{C} / \mathrm{m}^{2}$, indicating the same amount of accumulated negative charge on FEP surface. After 17 times of injection, the charge density was saturated at $\sim 630 \mu \mathrm{C} / \mathrm{m}^{2}$, which was the MSCD of this $50 \mu \mathrm{m}-$ thick FEP film. This increment of charge density was subsequently investigated by assembling 
the ion-injected FEP film into a conventional contact-separation TENG with an Al plate as the counter electrode. The short-circuit charge density $\left(\Delta \sigma_{S C}\right)$ generated by this TENG should equal to the surface charge density $\left(\sigma_{0}\right)$ on the FEP film. Figure 5c presented the recorded $\Delta \sigma_{S C}$ of TENG after each ion injection. With no injection, the initial $\Delta \sigma_{S C}$ was only about $50 \mu C / \mathrm{m}^{2}$. One ion injection process could increase $\Delta \sigma_{S C}$ to $\sim 100 \mu \mathrm{C} / \mathrm{m}^{2}$. After that, $\Delta \sigma_{S C}$ was elevated gradually by multiple ion injection processes and ultimately arrived at $\sim 240 \mu \mathrm{C} / \mathrm{m}^{2}$ at the fifth injection. Further increasing the ion injection times only resulted in very small amount of $\Delta \sigma_{\text {SC }}$ enhancement. After the ninth injection, the charge transfer suddenly behaved differently, as marked in the red dashed box. The first pressing process induced a $\Delta \sigma_{S C}$ of $\sim 260 \mu C / \mathrm{m}^{2}$. However, when Al was released and separated from FEP film, the $\Delta \sigma_{S C}$ dropped to $\sim 230 \mu C / \mathrm{m}^{2}$, leading to the upward shift of the base line. In the following contact and separation process, the $\Delta \sigma_{S C}$ was maintained at the same level. This abrupt decrease of $\Delta \sigma_{S C}$ was attributed to the air breakdown during the first releasing process, which was caused by a voltage drop between Al plate and FEP polymer. The presence of this voltage drop was verified by the numerically calculated potential distribution across the TENG (Figure 5d). As shown in the bottom portion of Figure 5d, a voltage drop $\left(V_{\text {gap }}\right)$ of $\sim 300 \mathrm{~V}$ existed in the air gap. When this $V_{\text {gap }}$ exceeded the breakdown voltage of air, the positive ions in the air discharging corona will partially screen the negative charge on the FEP surface, resulting in the reduced $\Delta \sigma_{S C}$.

As discussed above, the initial short-circuit charge density $\left(\Delta \sigma_{S C-I}\right)$ from the first pressing motion would be larger than that from the remaining operation circles $\left(\Delta \sigma_{S C-R}\right)$ after the ninth ion injection. Their relationship was summarized in Figure 5e, where two regions can be clearly recognized. At region I, the $V_{\text {gap }}$ caused by the FEP charge was smaller than the air breakdown 
voltage, where the $\Delta \sigma_{S C-R}$ was linearly increased with the enlargement of $\Delta \sigma_{S C-I}$. When $\Delta \sigma_{S C-I}$ reached the MSCD of $\sim 240 \mu \mathrm{C} / \mathrm{m}^{2}$, the $\Delta \sigma_{S C-R}$ started to deviate from $\Delta \sigma_{S C-I}$ due to the air breakdown (region II of Figure 5e). In this region, larger $\Delta \sigma_{S C-I}$ led to smaller $\Delta \sigma_{S C-R}$, implying that the discharging of the air was more intensive with higher $\Delta \sigma_{\text {SC-I. }}$.

During this ion injection process, the MSCD of triboelectric polymers can be measured. In this work, the relationship between the MSCD $\left(\sigma_{\max }\right)$ and film thickness $(d)$ of FEP was studied both theoretically and experimentally. As demonstrated by the calculated results in Figure $5 \mathrm{f}$ and $5 \mathrm{~g}$, when $d$ decreased from tens of microns to hundreds of nanometers, the MSCD was enhanced by more than two orders of magnitudes, approaching $50 \mathrm{mC} / \mathrm{m}^{2}$. Experimentally recorded MSCD of three FEP films with the thicknesses of 50, 75 and $125 \mu \mathrm{m}$ followed the same trend of the calculated curve (Figure 5g). The good agreement of experimental and simulated results confirmed the conclusion that a thinner dielectric film is favorable for the TENG development via the improvement of surface charge density, which was consistent with other report.[15]

In addition to the MSCD investigation, this ion injection approach can substantially enhance the output of TENG by maximizing the surface charge density. As shown in Figure 5c, ion injection could enable $\sim 5$ times larger charge density on FEP surface. In principle, proportionally-enhanced open circuit voltage $\left(V_{o c}\right)$ and short circuit current density $\left(J_{s c}\right)$ should be obtained accordingly. Figure 6 compared the $V_{o c}, J_{s c}$, and the power output of TENG before and after the ion injection. Without injection, the spontaneous charge density from the friction could only deliver a $V_{o c}$ of $\sim 200 \mathrm{~V}$ (Figure 6a). When the surface charge density was boosted to MSCD by ion injection, the $V_{o c}$ was increased to $\sim 1000 \mathrm{~V}$ (Figure 6b). Similarly, the $J_{s c}$ was increased from 18 to $78 \mathrm{~mA} / \mathrm{cm}^{2}$ via the ion injection under a pressing force of $\sim 20 \mathrm{~N}$ (Figures 6c 
and d). When deforming the TENG at $\sim 300 \mathrm{~N}$, the $J_{s c}$ could reach as high as $\sim 900 \mathrm{~mA} / \mathrm{cm}^{2}$ (Figure 6e). Under this testing condition, the actual power output of the ion-injected TENG was estimated by connecting the device with a series of loads with different resistances. As shown in Figure 6f, the voltage and current output increased and decreased gradually with the increase of the load resistance, respectively. The opposite trend of voltage and current output produced a maximum power output density of $\sim 315 \mathrm{~W} / \mathrm{m}^{2}$ with the external resistance of $300 \mathrm{M} \Omega$ (Figure 6g).

In general, the ion injection approach is simple, effective and applicable to many triboelectric polymers. It is a fairly efficient technique and by far the most commonly adopted method of polymer surface modification for improving TENGs' output. Nevertheless, the fabrication conditionsand processes are relatively complicated, which may bring obstacles for further scaling up, particularly considering the polymer sheets are extremely low-cost and roll-to-roll manufactured raw materials.

\section{Sequential Infiltration Synthesis}

Sequential infiltration synthesis (SIS) is a molecular infiltration process on the basis of atomic layer deposition (ALD) technique.[73-75] When certain polymers were implemented, the large permittivity of metalorganic ALD precursors allow the deep infiltration of inorganic compounds during ALD process, leading to inorganic/organic hybrid materials. SIS has been successfully used to convert the block co-polymer nanopatterns into more durable inorganic patterns and to improve the polymeric lithography resistance towards the subsequent etching.[76-78] Inspired by these developments, SIS was expected to be effective in tailoring the internal composition and 
electrical properties of polymer films, which may provide an ultimate solution for modifying triboelectric materials in bulk volume.

Based on SIS, $\mathrm{Yu}$ and coworkers reported an internal $\mathrm{AlO}_{\mathrm{x}}$ doping of several triboelectric polymers, including PDMS, polyimide (Kapton) and poly(methyl methacrylate) (PMMA).[79] $\mathrm{AlO}_{\mathrm{x}}$ molecules were found to be capable of penetrating into these polymers as deep as $\sim 3 \mu \mathrm{m}$, which effectively tuned the bulk electrical property of the films. TENG devices using the modified polymer films exhibited enhanced power output; and this enhancement remained effective after the surface of polymer film was polished off for more than $2 \mu \mathrm{m}$. $\mathrm{AlO}_{\mathrm{x}}$ was selected as the dopants since trimenthy aluminum (TMA, ALD precursor of $\mathrm{Al}_{2} \mathrm{O}_{3}$ ) has desirable permittivity in a number of polymers such as polystyrene (PS), PP, polyethylene (PE), and poly(vinyl chloride) (PVC) and PMMA.[73] The SIS doping process was carried out in a homemade ALD system by sequentially introducing TMA and $\mathrm{H}_{2} \mathrm{O}$ vapor to the polymer film at $80^{\circ} \mathrm{C}$ with controlled exposure time and cycles. Figures $7 \mathrm{a}$ and $\mathrm{b}$ show the cross-sectional SEM images and corresponding energy dispersive spectroscopy (EDS) elemental mappings of the pristine PDMS film and the same film after 5-cycle sequential TMA/ $\mathrm{H}_{2} \mathrm{O}$ infiltration (marked as $\mathrm{AlO}_{\mathrm{x}}$-doped PDMS), respectively. PDMS elements including silicon (Si), oxygen (O) and carbon (C) were all identified with a uniform distribution in the pristine film and no aluminum (Al) signal was detected (Figure 7a-ii-v). For the SIS-treated PDMS, Al signal was clearly detected and was concentrated within the top $\sim 3 \mu \mathrm{m}$ region, revealing the effective infiltration depth (Figure 7b-ii). Similarly, both O and C elements exhibited a concentrated signal within the top $\sim 3 \mu \mathrm{m}$ region matching well with $\mathrm{Al}$ distribution (Figure $7 \mathrm{~b}-\mathrm{iv}$ and $\mathrm{v}$ ). The concentrated $\mathrm{O}$ element was originated from Al-O bonds formed after $\mathrm{H}_{2} \mathrm{O}$ molecule infiltrated into polymers and reacted with TMA. The unreacted methyl groups on TMA could be the main source 
responsible for the concentrated $\mathrm{C}$ signal. This elemental distribution analysis evidences the successful deep infiltration of both precursors in the polymer film and no surface coating of $\mathrm{Al}_{2} \mathrm{O}_{3}$ was identified.

XPS characterizations further confirmed the presence of Al element in the PDMS after the SIS infiltration. As shown in figure 7c, a small peak of Al 2p appeared at the low binding energy region from the $\mathrm{AlO}_{\mathrm{x}}$-doped PDMS film. The high resolution scan of $\mathrm{Al} 2 \mathrm{p}$ signal demonstrated a peak shift from $75.6 \mathrm{eV}$ to $74.7 \mathrm{eV}$ after the SIS treatment, which was caused by the formation of the defective aluminum oxides such as $\mathrm{Al}_{2} \mathrm{O}$ and $\mathrm{AlO}$ (Figure 7d).[80] The nonstoichiometric aluminum oxide may introduce extra charge carrier sites and thus improve the charge capacitance of the modified PDMS film. This hypothesis was verified by the dielectric constant measurement (Figure 7e). SIS doping increased the dielectric constant of PDMS films from 2.4 to 2.7 within the test frequency range, implying that modified PDMS was capable of storing more charges compared with the pristine one.

Similar to previously discussed two strategies, the SIS doping could also arbitrarily tune the electron affinity of triboelectric polymers and modulate the charge attraction/repulsion ability. For the $\mathrm{AlO}_{\mathrm{x}}$ doping case, aluminum oxide will lower the electron affinity of PDMS since $\mathrm{AlO}_{\mathrm{x}}$ is a triboelectric positive material with high tendency to lose electrons. As a consequence, electron would be injected from $\mathrm{AlO}_{\mathrm{x}}$-doped PDMS to PDMS upon their frication, leading to a positively charged surface in the doped PDMS and negatively charged surface in the pristine PDMS (Figure 8a). On the contrary, two PDMS films without modification could not induce such a charge redistribution due to the identical surface potential. Therefore, the $\mathrm{AlO}_{\mathrm{x}}$ doping process should be able to significantly raise the TENG performance. Figures 8b-d show the $V_{o c}$, $J_{s c}$, and the total amount of charge transferred under short-circuit condition $\left(\Delta Q_{s c}\right)$ of TENGs 
with two configurations: PDMS-PDMS and PDMS-AlO ${ }_{\mathrm{x}}$-doped PDMS. For pair of PDMS$\mathrm{AlO}_{\mathrm{x}}$-doped PDMS, the average peak values of the $V_{o c}, J_{s c}$, and $\Delta Q_{s c}$ reached $2.3 \mathrm{~V}, 2.2 \mu \mathrm{A} / \mathrm{cm}^{2}$ and $45 \mu \mathrm{C} / \mathrm{m}^{2}$, respectively, which were comparable to TENGs that built with typical triboelectric couples such as teflon-metal pair.[15, 17] In comparison, only $0.3 \mathrm{~V}$ of $V_{o c}, 0.3$ $\mu \mathrm{A} / \mathrm{cm}^{2}$ of $J_{s c}$ and $5 \mu \mathrm{C} / \mathrm{m}^{2}$ of $\Delta Q_{s c}$ were obtained for the untreated PDMS pair.

One unique advantage of this SIS doping method is the simultaneous surface and bulk modification of the polymer's electrical property. The bulk doping capability allowed that the performance gain of TENGs could survive even after polishing off $2 \mu \mathrm{m}$-thick polymer materials. Figure 8e presents the $V_{o c}$ variation as a function of the removed thicknesses. $\sim 95 \%$ of $V_{o c}$ was remained after $2 \mu$ m-thick material was polished. Such a durable TENG performance was contributed by the deep penetration $(\sim 3 \mu \mathrm{m})$ of $\mathrm{AlO}_{\mathrm{x}}$ dopants. Further polishing the film dramatically reduced $V_{o c}$ due to the expose of unmodified PDMS. A minimum value of $\sim 0.3 \mathrm{~V}$ was received after removing $5 \mu$ m polymer, which was comparable to the TENG output with the pristine PDMS pair.

The up limit of this SIS doping process was revealed by varying the infiltration cycles and measuring the related TENGs output. As shown in Figure 8f, the peak $V_{o c}$ was improved from $0.3 \mathrm{~V}$ to $1.9 \mathrm{~V}$ by only 2 cycles of SIS, suggesting the high effectiveness of this polymer modification approach. The $V_{o c}$ kept increasing with the increment of SIS cycles and eventually saturated at $\sim 2.4 \mathrm{~V}$ after 10 cycles of infiltration. Almost stable values were recorded when further increasing the cycle numbers. This $V_{o c}$ variation is believed to be a consequence of the unique reaction process of $\mathrm{AlO}_{\mathrm{x}}$ in the polymer material. During the first 10 cycles of infiltration, TMA diffused into PDMS and accumulated inside the polymer film, resulting in the rapid 
increase of $V_{o c}$. After 10 cycles, more deposition might cover the entire PDMS surface and prevent further inside infiltration. Thus the growth mode switched back to regular ALD deposition of continuous $\mathrm{Al}_{2} \mathrm{O}_{3}$ film on the surface. Because additional cycles of infiltration contributed no internal doping, the electric property and TENG output would not exhibit any further change.

The underlying mechanism of this SIS strategy shares certain similarity with the aforementioned ion injection approaches. Both of them control the surface charge density of triboelectric polymer by introducing extra charged ions or molecules. However, compared to other chemical modification techniques, the SIS doping process extends the modification region from the surface to bulk portion of triboelectric polymers. This improvement is important for developing high-performance TENGs with good resistance to the surface wearing issue. Moreover, the SIS doping is versatile in tailoring broad range of triboelectric polymers since a variety of metalorganic molecules possess high permittivity in most polymer chains.[81] With proper selection of doping precursor and precise control of infiltration condition, SIS doping could be a promising strategy to engineering electric and dielectric properties of many polymers, and improving the performance of functional polymer based devices, such as TENGs.

\section{Molecular-Targeting Functionalization}

The chemical modification process through molecular-targeting functionalization is closely related to the chemical sensing function of TENGs. TENG is a good platform for developing various sensors including motion and chemical sensors.[46] By recording the output variation, TENG-based motion sensors can detect different types of mechanical deformations such as human touch and sound. Meanwhile, TENGs can also be used as self-powered chemical sensors 
since the amplitude of the triboelectric signal is proportional to the surface charge density that is largely influenced by the surface chemical state. The operation principle of the TENGs-based chemical sensor relies on the surface charge density variation induced by targeting molecules. When the target molecules are attached on the surface of triboelectric polymers, possible charge transfer could emerge between the chemical species and triboelectric materials, leading to the change of TENG output.[21, 82-84] In this section, the TENG-based chemical sensors are discussed with an emphasis on surface functionalization for detecting specific molecules.

Li et al. reported a self-powered phenol sensor based on the $\beta$-cyclodextrin ( $\beta$-CD) enhanced triboelectrification process.[85] Figure 9a presents the schematic of the TENG device with PTFE and $\beta$-CD decorated $\mathrm{TiO}_{2} \mathrm{NWs}$ as two electrodes. $\mathrm{TiO}_{2} \mathrm{NWs}$ were grown on $\mathrm{Ti}$ foil with an average diameter and length of $73.4 \mathrm{~nm}$ and $1.92 \mu \mathrm{m}$. In this structure, $\beta$-CD was a bifunctional component that can recognize phenol molecule and enhance the TENG performance at the same time. On one hand, $\beta$-CD molecule is composed of a hydrophobic internal cavity and hydrophilic external side chains. This special structure allows it to capture guest molecules with appropriate polarity and dimension, and forming a host/guest inclusion complex. The diameter of $\beta-C D$ cavity is particularly suitable for the selective absorption of phenol, which making it a particular molecular probe for phenol.[86] On the other hand, $\beta$-CD can alter the overall TENG performance by conducting charge transfer with $\mathrm{TiO}_{2} \mathrm{NWs}$. Specifically, $\beta$-CD is binding on $\mathrm{TiO}_{2}$ surface via hydroxyl groups. Electrons could thus be injected from the hydroxyl groups to $\mathrm{TiO}_{2}$ surface due to the coordination effect between the ligand and the metal under visible light (Figure 9b).[87] Consequently, the positive charges on $\mathrm{TiO}_{2}$ surface that produced from the triboelectrification with PTFE were partially neutralized. This means PFTE can extract extra electrons from $\mathrm{TiO}_{2}$ after $\beta$-CD functionalization, and further increase its surface charge density, 
and therefore improve the TENG output. The influences of $\beta$-CD concentration on the triboelectric output were studied with a $4 \mathrm{~cm} \times 4 \mathrm{~cm}$ TENG (Figure 9c). Both current and voltage signals were proportionally increased with the augment of $\beta$-CD concentration (Figure 9d). When TENG was treated with $80 \mathrm{mM} \beta-\mathrm{CD}$, the electric output reached $\sim 55 \mathrm{~V}$, which was more than 8 times larger than the unmodified devices. A voltage plateau emerged at the region of $\beta$ $\mathrm{CD}$ concentration larger than $80 \mathrm{mM}$, which may be caused by the saturation of $\beta-\mathrm{CD}$ on $\mathrm{TiO}_{2}$ surface. This experiment strongly evidenced the TENG performance-gain function of the $\beta$-CD modification.

The phenol detecting ability of this $\beta$-CD tailored triboelectrification was evaluated on TENGs modified by a fixed $\beta$-CD concentration of $80 \mathrm{mM}$. The constant volumes $(20 \mathrm{~mL})$ of phenol solution with varied concentrations were introduced to treat the $\beta$-CD decorated $\mathrm{TiO}_{2}$ NWs. As shown in Figure 9e, the current output was clearly dependent on the phenol concentration. At the region of $10-100 \mathrm{mM}$ phenol, the current signal decreased almost linearly with the increase of the phenol concentration. The reduction of electric output is ascribed to the phenol absorption onto the $\mathrm{TiO}_{2} \mathrm{NWs}$ surface via the $\beta$-CD cavity. After the whole surface was covered by phenol, the triboelectrification process might only happen between PTFE and phenol instead of the original PTFE and $\mathrm{TiO}_{2}$. Whereas, compared with $\mathrm{TiO}_{2}$, phenol has lower tendency to give electron to PTFE, and therefore jeopardized the TENG output. To render a direct view of the detecting efficiency, the current ratio $\left(\left(\mathrm{I}_{0}-\mathrm{I}\right) / \mathrm{I}_{0}\right)$ was plotted as a function of phenol concentrations, where $\mathrm{I}_{0}$ was the original current output and I was the current output after phenol treatment. As shown in Figure 9f, the sensitivity of the TENG sensor was calculated to be $0.01 \mu \mathrm{M}^{-1}$ in the sensing range of $10-100 \mathrm{mM}$. 
Besides the good sensitivity, this $\beta$-CD modified TENG sensor exhibited excellent selectivity and reusability. The detecting performance of this sensor for a number of other organic species that possessed similar molecular structures with phenol were tested and presented in Figure 9g. With the same concentration of $50 \mathrm{mM}$, the recorded current ratio from phenol detecting was appreciably larger than all other organic species, implying the good selectivity of this sensor. Additionally, after the sensing process, the device could be completely recovered by ethyl alcohol rinsing without losing any electric output. The exceptional sensitivity, selectivity, reusability, low cost, environmentally friendly and self-powered characteristics promise this $\beta$ CD modified TENG in future phenol sensing field.

Jie et al. developed a self-powered TENG sensor for dopamine (DA) detection with PTFE-Al triboelectric pair. [88] The operation of this DA sensor relies on the chemical modification of PTFE with the polydopamine(PDA). Under slightly alkaline condition, DA molecule would go through a self-polymerization and produced PDA chains on the PTFE surfaces (Figure 10a). Figure 10b schematically depicts the PDA modification process, where PTFE film was first soaked in the buffer solution containing different concentrations of dopamine. After adjusting the PH to 8.5, considerable amount of PDA was formed on the PTFE surface, turning the film color from white to brown and increased the hydrophobicity of PTFE. Since the electron affinity of PDA layer was substantially smaller than PTFE, the PDA layer would significantly reduce the surface charge density of the PTFE film, and therefore reduced the TENG output.

Figure 10c manifests the current output ratio of TENG under a series of DA concentrations. In general, current output diminished with the increase of DA concentration. As shown in the inset of Figure 10c, a proximately linear relationship was identified between the current ratio and Napierian logarithm of the DA concentration within the testing range (10 $\mu \mathrm{M}$ and $1 \mathrm{mM})$. The 
detection limit of this TENG sensor was found to be $0.5 \mu \mathrm{M}$, indicating its good sensitivity. The selectivity of the TENG sensor was further studied by purposely introducing other interference factors into the DA solution including uric acid (UA) and ascorbic acid (AA). Conventional

electrochemical detections of DA usually suffer from the fouling effect resulted from the oxidation of UA and AA. However, in this TENG sensor, UA and AA can be well excluded since these two molecules have poor interaction between PTFE. Figure 10d compares the current ratio of the TENG sensor after exposing to DA, UA and AA solution with same concentration. As expected, only DA considerably altered the current signals, suggesting the good selectivity of this sensor.

Compared toconventional chemical monitoring techniques, TENG-based chemical sensors share common characteristics such as high sensitivity, selectivity and reusability. Meanwhile, since polymers are typically the active materials in TENG, existing principles of molecule detection relying on polymer reactions can be seamlessly transferred to TENG sensors. Most importantly, the self-powered capability of TENG-based chemical sensors greatly promise them in developing battery-free sensing systems with exceptional long life span.

\section{Conclusion}

Chemical modifications have been evidenced to be an effective approach for controlling the TENG output through regulating the surface charge density of triboelectric polymers. By enhancing the surface charge density, numerous polymers can be utilized to build highperformance TENGs without losing their intrinsic merits. The largely broadened material selections allow more flexible structural designs of TENGs. Compared to the vast research efforts of pursuing new configurations and functions, chemical properties and surface 
functionalization of triboelectric polymers are relatively less explored despite their fundamental role in controlling the charge density and overall triboelectric performance. Both theoretical calculations and experimental endeavors are highly desired for promoting the existing or discovering new chemical modification techniques. Computational predictions would facilitate our understanding of surface chemical states and consequently guide experimental explorations. Developing innovative chemical functionalizing process for the teflon family can potentially uplift the limit of TENG outputs. Chemical modification, particularly to the bulk properties, would bring practical impacts to output enhancement, device configuration optimization and working lifetime extension of commercial TENG devices in the near future.

Acknowledgements: The authors thank the support of National Science Foundation under Award \# CMMI-1148919, US; and the "Thousands talents” program for pioneer researcher and his innovation team, China.

\section{Reference:}

[1] Z. L. Wang, Triboelectric nanogenerators as new energy technology for self-powered systems and as active mechanical and chemical sensors, ACS Nano7 (2013) 9533-9557.

[2] Z. L. Wang; J. Chen; L. Lin, Progress in triboelectric nanogenerators as a new energy technology and self-powered sensors, Energy Environ. Sci.8 (2015) 2250-2282.

[3] Z. L. Wang; J. Song, Piezoelectric nanogenerators based on zinc oxide nanowire arrays, Science312 (2006) 242-246.

[4] Y. Mao; P. Zhao; G. McConohy; H. Yang; Y. Tong; X. Wang, Sponge-like piezoelectric polymer films for scalable and integratable nanogenerators and self-powered electronic systems, Adv. Energy Mater.4 (2014) 130624.

[5] K. I. Park; M. Lee; Y. Liu; S. Moon; G. T. Hwang; G. Zhu; J. E. Kim; S. O. Kim; K. Kim do; Z. L. Wang; K. J. Lee, Flexible nanocomposite generator made of $\mathrm{BaTiO}_{3}$ nanoparticles and graphitic carbons, Adv. Mater.24 (2012) 2999-3004.

[6] X. Wang; J. Song; J. Liu; Z. L. Wang, Direct-current nanogenerator driven by ultrasonic waves, Science316 (2007) 102-105. 
[7] S. N. Cha; J. S. Seo; S. M. Kim; H. J. Kim; Y. J. Park; S. W. Kim; J. M. Kim, Sound-driven piezoelectric nanowire-based nanogenerators, Adv. Mater.22 (2010) 4726-4730.

[8] X. Wang, Piezoelectric nanogenerators-harvesting ambient mechanical energy at the nanometer scale, Nano Energy1 (2012) 13-24.

[9] J.-H. Lee; K. Y. Lee; B. Kumar; N. T. Tien; N.-E. Lee; S.-W. Kim, Highly sensitive stretchable transparent piezoelectric nanogenerators, Energy Environ. Sci.6 (2013) 169-175.

[10] X. Wang; J. Liu; J. Song; Z. L. Wang, Integrated nanogenerators in biofluid, Nano Lett.7 (2007) 2475-2479.

[11] Y. Qin; X. Wang; Z. L. Wang, Microfibre-nanowire hybrid structure for energy scavenging, Nature451 (2008) 809-813.

[12]C. Sun; J. Shi; D. J. Bayerl; X. Wang, PVDF microbelts for harvesting energy from respiration, Energy Environ. Sci.4 (2011) 4508-4512.

[13]M. Lee; C. Y. Chen; S. Wang; S. N. Cha; Y. J. Park; J. M. Kim; L. J. Chou; Z. L. Wang, A hybrid piezoelectric structure for wearable nanogenerators, Adv. Mater.24 (2012) 1759-1764.

[14]F.-R. Fan; Z.-Q. Tian; Z. Lin Wang, Flexible triboelectric generator, Nano Energy1 (2012) 328-334.

[15]G. Zhu; Y. S. Zhou; P. Bai; X. S. Meng; Q. Jing; J. Chen; Z. L. Wang, A shape-adaptive thin-film-based approach for 50\% high-efficiency energy generation through micro-grating sliding electrification, Adv. Mater.26 (2014) 3788-3796.

[16]W. Tang; T. Jiang; F. R. Fan; A. F. Yu; C. Zhang; X. Cao; Z. L. Wang, Liquid-metal electrode for high-performance triboelectric nanogenerator at an instantaneous energy conversion efficiency of 70.6\%, Adv. Funct. Mater.25 (2015) 3718-3725.

[17]Y. Xie; S. Wang; S. Niu; L. Lin; Q. Jing; J. Yang; Z. Wu; Z. L. Wang, Grating-structured freestanding triboelectric-layer nanogenerator for harvesting mechanical energy at $85 \%$ total conversion efficiency, Adv. Mater.26 (2014) 6599-6607.

[18]S. Wang; L. Lin; Z. L. Wang, Nanoscale triboelectric-effect-enabled energy conversion for sustainably powering portable electronics, Nano Lett.12 (2012) 6339-6346.

[19]G. Zhu; J. Chen; T. Zhang; Q. Jing; Z. L. Wang, Radial-arrayed rotary electrification for high performance triboelectric generator, Nat. Commun.5 (2014) 3426.

[20]G. Zhu; W. Q. Yang; T. Zhang; Q. Jing; J. Chen; Y. S. Zhou; P. Bai; Z. L. Wang, Selfpowered, ultrasensitive, flexible tactile sensors based on contact electrification, Nano Lett.14 (2014) 3208-3213.

[21]Z. Wen; J. Chen; M.-H. Yeh; H. Guo; Z. Li; X. Fan; T. Zhang; L. Zhu; Z. L. Wang, Blowdriven triboelectric nanogenerator as an active alcohol breath analyzer, Nano Energy16 (2015) 38-46.

[22]Q. Zheng; B. Shi; F. Fan; X. Wang; L. Yan; W. Yuan; S. Wang; H. Liu; Z. Li; Z. L. Wang, In vivo powering of pacemaker by breathing-driven implanted triboelectric nanogenerator, Adv. Mater.26 (2014) 5851-5856. 
[23]J. Yang; J. Chen; Y. Su; Q. Jing; Z. Li; F. Yi; X. Wen; Z. Wang; Z. L. Wang, Eardruminspired active sensors for self-powered cardiovascular system characterization and throatattached anti-interference voice recognition, Adv. Mater.27 (2015) 1316-1326.

[24]R. Zhang; S. Wang; M. H. Yeh; C. Pan; L. Lin; R. Yu; Y. Zhang; L. Zheng; Z. Jiao; Z. L. Wang, A streaming potential/current-based microfluidic direct current generator for selfpowered nanosystems, Adv. Mater. 27(2015) 6482-6487.

[25]P. K. Yang; L. Lin; F. Yi; X. Li; K. C. Pradel; Y. Zi; C. I. Wu; J. H. He; Y. Zhang; Z. L. Wang, A flexible, stretchable and shape-adaptive approach for versatile energy conversion and self-powered biomedical monitoring, Adv. Mater.27 (2015) 3817-3824.

[26]J. Chen; G. Zhu; J. Yang; Q. Jing; P. Bai; W. Yang; X. Qi; Y. Su; Z. L. Wang, Personalized keystroke dynamics for self-powered human-machine interfacing, ACS Nano9 (2015) 105116.

[27] X. Wang; S. Wang; Y. Yang; Z. L. Wang, Hybridized electromagnetic-triboelectric nanogenerator for scavenging air-flow energy to sustainably power temperature sensors, ACS Nano9 (2015) 4553-4562.

[28] X. Fan; J. Chen; J. Yang; P. Bai; Z. Li; Z. L. Wang, Ultrathin, rollable, paper-based triboelectric nanogenerator for acoustic energy harvesting and self-powered sound recording, ACS Nano9 (2015) 4236-4243.

[29] M. Kanik; M. G. Say; B. Daglar; A. F. Yavuz; M. H. Dolas; M. M. El-Ashry; M. Bayindir, A motion- and sound-activated, 3D-printed, chalcogenide-based triboelectric nanogenerator, Adv. Mater.27 (2015) 2367-2376.

[30] Z. H. Lin; G. Cheng; S. Lee; K. C. Pradel; Z. L. Wang, Harvesting water drop energy by a sequential contact-electrification and electrostatic-induction process, Adv. Mater.26 (2014) 4690-4696.

[31] J. Chun; J. W. Kim; W.-s. Jung; C.-Y. Kang; S.-W. Kim; Z. L. Wang; J. M. Baik, Mesoporous pores impregnated with Au nanoparticles as effective dielectrics for enhancing triboelectric nanogenerator performance in harsh environments, Energy Environ. Sci.8 (2015) 3006-3012.

[32] Y. Mao; D. Geng; E. Liang; X. Wang, Single-electrode triboelectric nanogenerator for scavenging friction energy from rolling tires, Nano Energy15 (2015) 227-234.

[33] J. M. Wu; C. K. Chang; Y. T. Chang, High-output current density of the triboelectric nanogenerator made from recycling rice husks, Nano Energy19 (2016) 39-47.

[34] Y. Yang; G. Zhu; H. Zhang; J. Chen; X. Zhong; Z. H. Lin; Y. Su; P. Bai; X. Wen; Z. L. Wang, Triboelectric nanogenerator for harvesting wind energy and as self-powered wind vector sensor system, ACS Nano7 (2013) 9461-9468.

[35] Z. L. Wang, Triboelectric nanogenerators as new energy technology and self-powered sensors-principles, problems and perspectives, Faraday Discuss.176 (2014) 447-458.

[36] X. Wang; S. Niu; Y. Yin; F. Yi; Z. You; Z. L. Wang, Triboelectric nanogenerator based on fully enclosed rolling spherical structure for harvesting low-frequency water wave energy, Adv. Energy Mater. 5(2015) 1501467. 
[37] J. Chen; J. Yang; Z. Li; X. Fan; Y. Zi; Q. Jing; H. Guo; Z. Wen; K. C. Pradel; S. Niu; Z. L. Wang, Networks of triboelectric nanogenerators for harvesting water wave energy: a potential approach toward blue energy, ACS Nano9 (2015) 3324-3331.

[38] G. Zhu; Y. Su; P. Bai; J. Chen; Q. Jing; W. Yang; Z. L. Wang, Harvesting water wave energy by asymmetric screening of electrostatic charges on a nanostructured hydrophobic thin-film surface, ACS Nano8 (2014) 6031-6037.

[39] S. Wang; X. Mu; X. Wang; A. Y. Gu; Z. L. Wang; Y. Yang, Elasto-aerodynamics-driven triboelectric nanogenerator for scavenging air-flow energy, ACS Nano9 (2015) 9554-9563.

[40] Y. Xie; S. Wang; L. Lin; Q. Jing; Z. H. Lin; S. Niu; Z. Wu; Z. L. Wang, Rotary triboelectric nanogenerator based on a hybridized mechanism for harvesting wind energy, ACS Nano7 (2013) 7119-7125.

[41] H. R. Zhu; W. Tang; C. Z. Gao; Y. Han; T. Li; X. Cao; Z. L. Wang, Self-powered metal surface anti-corrosion protection using energy harvested from rain drops and wind, Nano Energy14 (2015) 193-200.

[42] H. Zhang; Y. Yang; X. Zhong; Y. Su; Y. Zhou; C. Hu; Z. L. Wang, Single-electrode-based rotating triboelectric nanogenerator for harvesting energy from tires, ACS Nano8 (2014) 680689.

[43] S. Wang; Y. Xie; S. Niu; L. Lin; Z. L. Wang, Freestanding triboelectric-layer-based nanogenerators for harvesting energy from a moving object or human motion in contact and non-contact modes, Adv. Mater.26 (2014) 2818-2824.

[44] G. Zhu; P. Bai; J. Chen; Z. Lin Wang, Power-generating shoe insole based on triboelectric nanogenerators for self-powered consumer electronics, Nano Energy2 (2013) 688-692.

[45] G. Zhu; B. Peng; J. Chen; Q. Jing; Z. Lin Wang, Triboelectric nanogenerators as a new energy technology: From fundamentals, devices, to applications, Nano Energy14 (2015) 126138.

[46] S. Wang; L. Lin; Z. L. Wang, Triboelectric nanogenerators as self-powered active sensors, Nano Energy11 (2015) 436-462.

[47] X.-S. Zhang; M.-D. Han; B. Meng; H.-X. Zhang, High performance triboelectric nanogenerators based on large-scale mass-fabrication technologies, Nano Energy11 (2015) 304-322.

[48] R. Hinchet; W. Seung; S. W. Kim, Recent Progress on Flexible Triboelectric Nanogenerators for Self-Powered Electronics, ChemSusChem8 (2015) 2327-2344.

[49] L. Zheng; Z.-H. Lin; G. Cheng; W. Wu; X. Wen; S. Lee; Z. L. Wang, Silicon-based hybrid cell for harvesting solar energy and raindrop electrostatic energy, Nano Energy9 (2014) 291300.

[50] L. Lin; Y. Xie; S. Niu; S. Wang; P. K. Yang; Z. L. Wang, Robust triboelectric nanogenerator based on rolling electrification and electrostatic induction at an instantaneous energy conversion efficiency of approximately 55\%, ACS Nano9 (2015) 922-930. 
[51] W. Seung; M. K. Gupta; K. Y. Lee; K. S. Shin; J. H. Lee; T. Y. Kim; S. Kim; J. Lin; J. H. Kim; S. W. Kim, Nanopatterned textile-based wearable triboelectric nanogenerator, ACS Nano9 (2015) 3501-3509.

[52] G. Zhu; Z. H. Lin; Q. Jing; P. Bai; C. Pan; Y. Yang; Y. Zhou; Z. L. Wang, Toward largescale energy harvesting by a nanoparticle-enhanced triboelectric nanogenerator, Nano Lett.13 (2013) 847-853.

[53] B. Dudem; Y. H. Ko; J. W. Leem; S. H. Lee; J. S. Yu, Highly transparent and flexible triboelectric nanogenerators with subwavelength-architectured polydimethylsiloxane by a nanoporous anodic aluminum oxide Template, ACS Appl. Mater. Interfaces7 (2015) 2052020529.

[54] D. Choi; D. Yoo; D. S. Kim, One-step fabrication of transparent and flexible nanotopographical-triboelectric nanogenerators via thermal nanoimprinting of thermoplastic fluoropolymers, Adv. Mater.27 (2015) 7386-7394.

[55] X. S. Zhang; M. D. Han; R. X. Wang; F. Y. Zhu; Z. H. Li; W. Wang; H. X. Zhang, Frequency-multiplication high-output triboelectric nanogenerator for sustainably powering biomedical microsystems, Nano Lett.13 (2013) 1168-1172.

[56] F. R. Fan; L. Lin; G. Zhu; W. Wu; R. Zhang; Z. L. Wang, Transparent triboelectric nanogenerators and self-powered pressure sensors based on micropatterned plastic films, Nano letters12 (2012) 3109-3114.

[57] C. K. Jeong; K. M. Baek; S. Niu; T. W. Nam; Y. H. Hur; D. Y. Park; G. T. Hwang; M. Byun; Z. L. Wang; Y. S. Jung; K. J. Lee, Topographically-designed triboelectric nanogenerator via block copolymer self-assembly, Nano Lett.14 (2014) 7031-7038.

[58] D. Kim; S.-B. Jeon; J. Y. Kim; M.-L. Seol; S. O. Kim; Y.-K. Choi, High-performance nanopattern triboelectric generator by block copolymer lithography, Nano Energy12 (2015) 331-338.

[59] K. Y. Lee; J. Chun; J. H. Lee; K. N. Kim; N. R. Kang; J. Y. Kim; M. H. Kim; K. S. Shin; M. K. Gupta; J. M. Baik; S. W. Kim, Hydrophobic sponge structure-based triboelectric nanogenerator, Adv. Mater.26 (2014) 5037-42.

[60] A. F. Diaz; R. M. Felix-Navarro, A semi-quantitative tribo-electric series for polymeric materials: the influence of chemical structure and properties, J. Electrost.62 (2004) 277-290.

[61] S. H. Shin; Y. H. Kwon; Y. H. Kim; J. Y. Jung; M. H. Lee; J. Nah, Triboelectric charging sequence induced by surface functionalization as a method to fabricate high performance triboelectric generators, ACS Nano9 (2015) 4621-4627.

[62] Y. Feng; Y. Zheng; S. Ma; D. Wang; F. Zhou; W. Liu, High output polypropylene nanowire array triboelectric nanogenerator through surface structural control and chemical modification, Nano Energy19 (2016) 48-57.

[63] X.-S. Zhang; M.-D. Han; R.-X. Wang; B. Meng; F.-Y. Zhu; X.-M. Sun; W. Hu; W. Wang; Z.-H. Li; H.-X. Zhang, High-performance triboelectric nanogenerator with enhanced energy density based on single-step fluorocarbon plasma treatment, Nano Energy4 (2014) 123-131. 
[64] X. Cheng; B. Meng; X. Chen; M. Han; H. Chen; Z. Su; M. Shi; H. Zhang, Single-step fluorocarbon plasma treatment-induced wrinkle structure for high-performance triboelectric nanogenerator, Small(2015) DOI:10.1002/smll.201502720.

[65] H. Y. Li; L. Su; S. Y. Kuang; C. F. Pan; G. Zhu; Z. L. Wang, Significant enhancement of triboelectric charge density by fluorinated surface modification in nanoscale for converting mechanical energy, Adv. Funct. Mater.25 (2015) 5691-5697.

[66] E. P. Ivanova; J. P. Wright; D. Pham; L. Filipponi; A. Viezzoli; D. V. Nicolau, Polymer microstructures fabricated via laser ablation used for multianalyte protein microassay, Langmuir18 (2002) 9539-9546.

[67] S. Raoux; T. Tanaka; M. Bhan; H. Ponnekanti; M. Seamons; T. Deacon; L. Q. Xia; F. Pham; D. Silvetti; D. Cheung; K. Fairbairn; A. Jonhson; R. Pearce; J. Langan, Remote microwave plasma source for cleaning chemical vapor deposition chambers: technology for reducing global warming gas emissions, J. Vac. Sci. Technol., B: Microelectron. Nanometer Struct.17 (1999) 477.

[68] P. M. Scott, $\mathrm{CF}_{4} / \mathrm{O}_{2}$ plasma etching and surface modification of polyimide films: Timedependent surface fluorination and fluorination model, J. Vac. Sci. Technol., A8 (1990) 2382.

[69] X. Wei; C. Xiaodong; W. Jianqi, Characterization of PET surface modification with $\mathrm{CF}_{4^{-}}$ $\mathrm{CH}_{4}\left(\mathrm{CF}_{4} / \mathrm{CH}_{4}>5\right) \mathrm{RF}$ plasma by XPS and contact angle techniques, J. Polym. Sci., Part A: Polym. Chem.33 (1995) 807-813.

[70] S. Wang; Y. Xie; S. Niu; L. Lin; C. Liu; Y. S. Zhou; Z. L. Wang, Maximum surface charge density for triboelectric nanogenerators achieved by ionized-air injection: methodology and theoretical understanding, Adv. Mater.26 (2014) 6720-6728.

[71] H. Fang; W. Wu; J. Song; Z. L. Wang, Controlled growth of aligned polymer nanowires, J. Phys. Chem. C 113 (2009) 16571-16574.

[72] H. O. Jacobs; G. M. Whitesides, Submicrometer patterning of charge in thin-film electrets, Science291 (2001) 1763-1766.

[73] C. A. Wilson; R. K. Grubbs; S. M. George, Nucleation and growth during $\mathrm{Al}_{2} \mathrm{O}_{3}$ atomic layer deposition on polymers, Chem. Mater.17 (2005) 5625-5634.

[74] M. Biswas; J. A. Libera; S. B. Darling; J. W. Elam, Kinetics for the sequential infiltration synthesis of alumina in poly(methyl methacrylate): an infrared spectroscopic study, J. Phys. Chem. C119 (2015) 14585-14592.

[75] Q. Peng; Y. C. Tseng; S. B. Darling; J. W. Elam, A route to nanoscopic materials via sequential infiltration synthesis on block copolymer templates, ACS Nano5 (2011) 46004606.

[76] Q. Peng; Y. C. Tseng; S. B. Darling; J. W. Elam, Nanoscopic patterned materials with tunable dimensions via atomic layer deposition on block copolymers, Adv. Mater.22 (2010) 5129-5133.

[77] Y.-C. Tseng; Q. Peng; L. E. Ocola; J. W. Elam; S. B. Darling, Enhanced block copolymer lithography using sequential infiltration synthesis, J. Phys. Chem. C115 (2011) 17725-17729. 
[78] R. Saberi Moghaddam; S. Huettner; Y. Vaynzof; C. Ducati; G. Divitini; R. H. Lohwasser; K. P. Musselman; A. Sepe; M. R. Scherer; M. Thelakkat; U. Steiner; R. H. Friend, Polymer crystallization as a tool to pattern hybrid nanostructures: growth of $12 \mathrm{~nm} \mathrm{ZnO}$ arrays in poly(3-hexylthiophene), Nano Lett.13 (2013) 4499-4504.

[79]Y. Yu; Z. Li; Y. Wang; S. Gong; X. Wang, Sequential infiltration synthesis of doped polymer films with tunable electrical properties for efficient triboelectric nanogenerator development, Adv. Mater.27 (2015) 4938-4944.

[80] H.-S. Kang; M. Siva Pratap Reddy; D.-S. Kim; K.-W. Kim; J.-B. Ha; Y. S. Lee; H.-C. Choi; J.-H. Lee, Effect of oxygen species on the positive flat-band voltage shift in $\mathrm{Al}_{2} \mathrm{O}_{3} / \mathrm{GaN}$ metal-insulator-semiconductor capacitors with post-deposition annealing, J. Phys. D: Appl. Phys.46 (2013) 155101.

[81] S. M. George, Atomic layer deposition: an overview, Chem. Rev.110 (2010) 111-131.

[82] J.-H. Kim; J. Chun; J. W. Kim; W. J. Choi; J. M. Baik, Self-powered, room-temperature electronic nose based on triboelectrification and heterogeneous catalytic reaction, Adv. Funct. Mater.25 (2015) 7049-7055.

[83] Z. H. Lin; G. Zhu; Y. S. Zhou; Y. Yang; P. Bai; J. Chen; Z. L. Wang, A self-powered triboelectric nanosensor for mercury ion detection, Angew. Chem.52 (2013) 5065-5069.

[84] Z. H. Lin; G. Cheng; W. Wu; K. C. Pradel; Z. L. Wang, Dual-mode triboelectric nanogenerator for harvesting water energy and as a self-powered ethanol nanosensor, ACS Nano8 (2014) 6440-6448.

[85] Z. Li; J. Chen; J. Yang; Y. Su; X. Fan; Y. Wu; C. Yu; Z. L. Wang, $\beta$-cyclodextrin enhanced triboelectrification for self-powered phenol detection and electrochemical degradation, Energy Environ. Sci.8 (2015) 887-896.

[86] X. Chen; X. Cheng; J. J. Gooding, Detection of trace nitroaromatic isomers using indium tin oxide electrodes modified using beta-cyclodextrin and silver nanoparticles, Anal. Chem.84 (2012) 8557-8563.

[87] Y. Zhou; M. Antonietti, Synthesis of very small $\mathrm{TiO}_{2}$ nanocrystals in a room-temperature ionic liquid and their self-assembly toward mesoporous spherical aggregates, J. Am. Chem. Soc.125 (2003) 14960-14961.

[88]Y. Jie; N. Wang; X. Cao; Y. Xu; T. Li; X. Zhang; Z. L. Wang, Self-powered triboelectric nanosensor with poly(tetrafluoroethylene) nanoparticle arrays for dopamine detection, ACS Nano9 (2015) 8376-8383. 
Figures and Captions

a
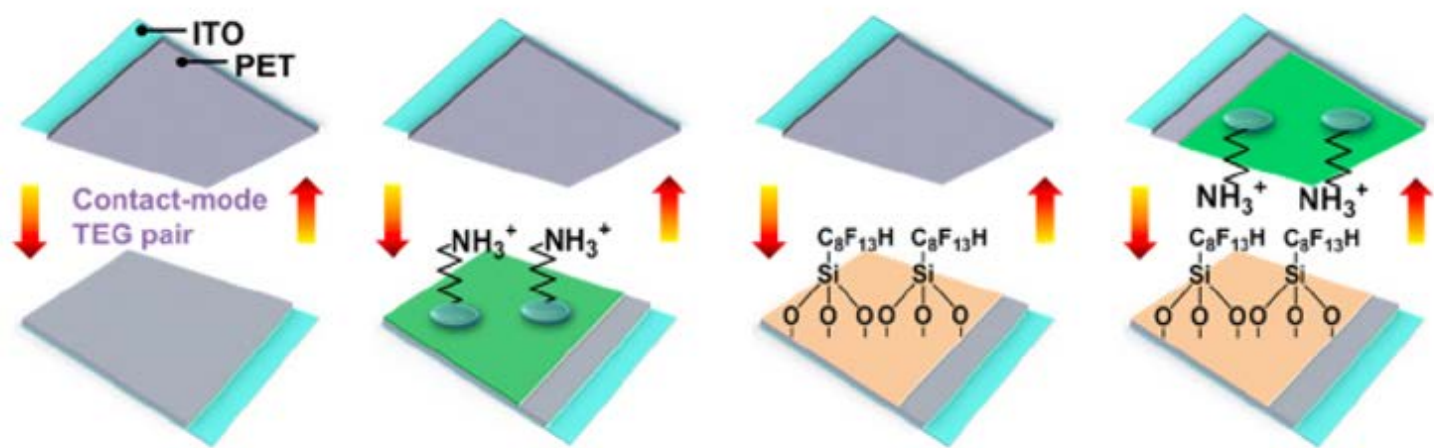

(i) PET : PET

(ii) PET : Poly-_-lysine

(iii) PET : FOTS

(iv) Poly-z-lysine : FOTS
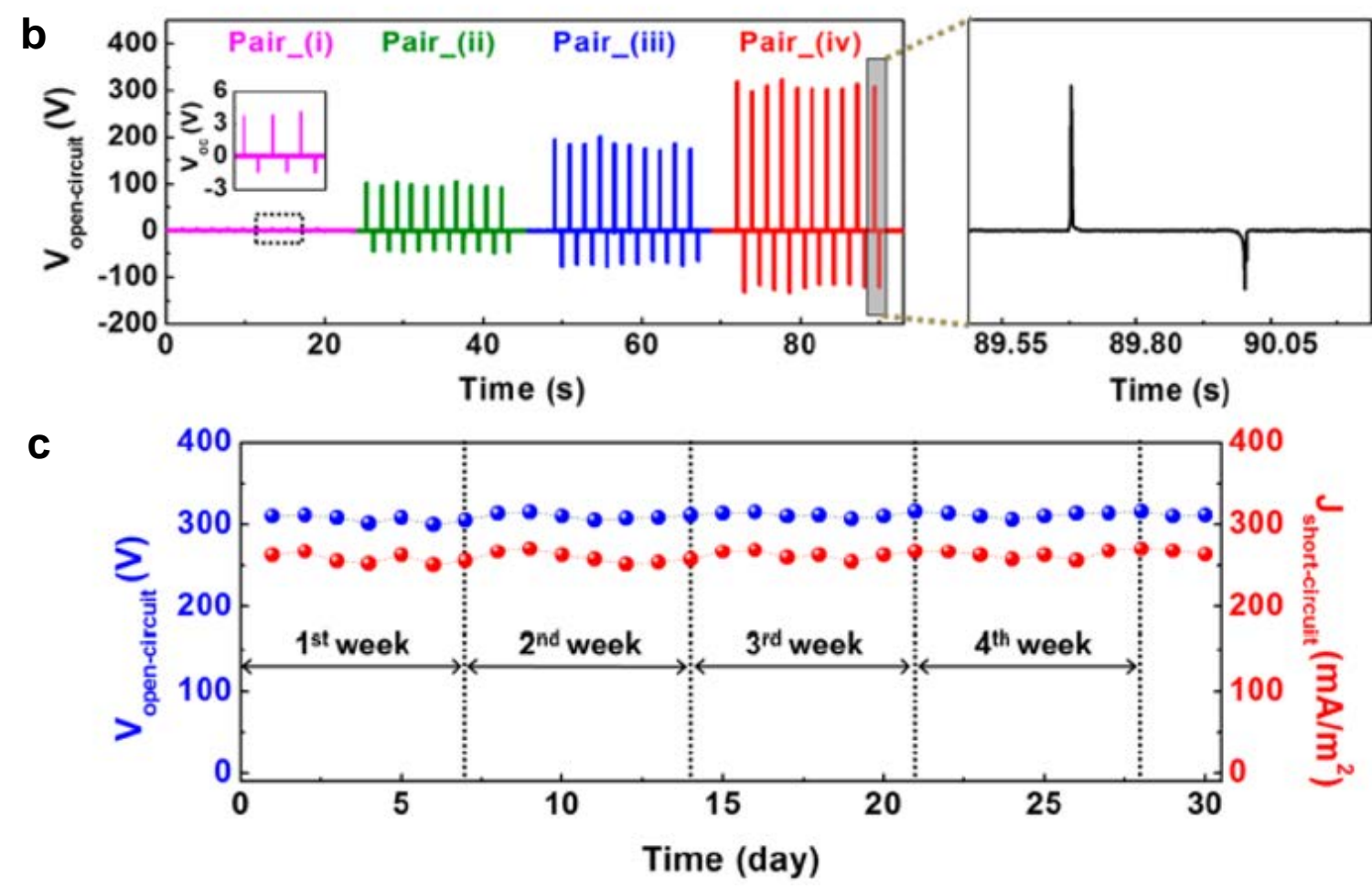
Figure 1. (a) Schematic of TENGs built with four different contact pairs: (i) PET:PET, (ii) PET:P-PET, (iii) PET:F-PET, (iv) P-PET:F-PET. (b) Open-circuit voltage $\left(V_{o c}\right)$ of corresponding TENGs, showing substantial performance gain after the chemical modifications. (c) Stability test of TENG with pair (iv), presenting no output reduction within one month. Reprinted with permission from ref 61. Copyright 2015, American Chemical Society.

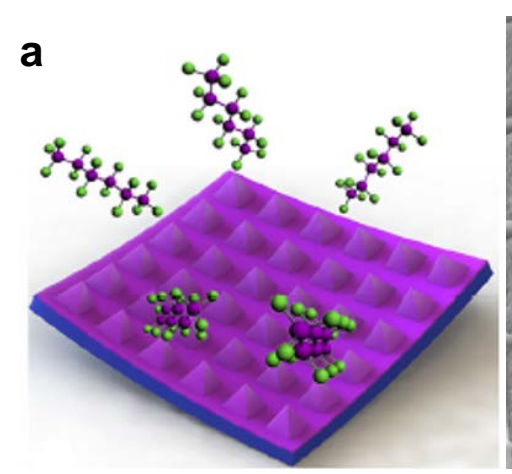

d

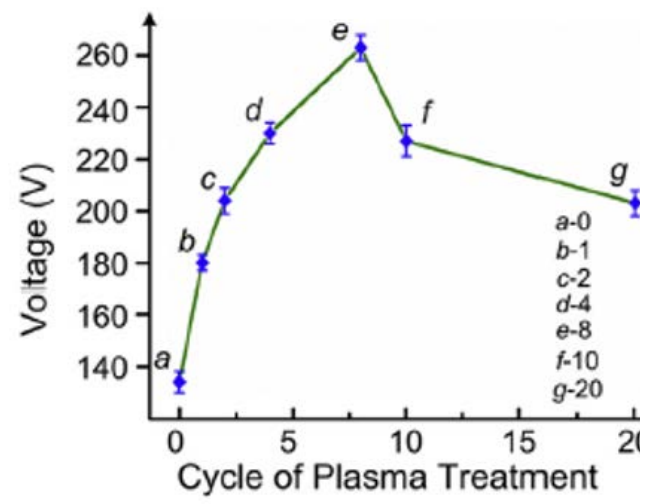

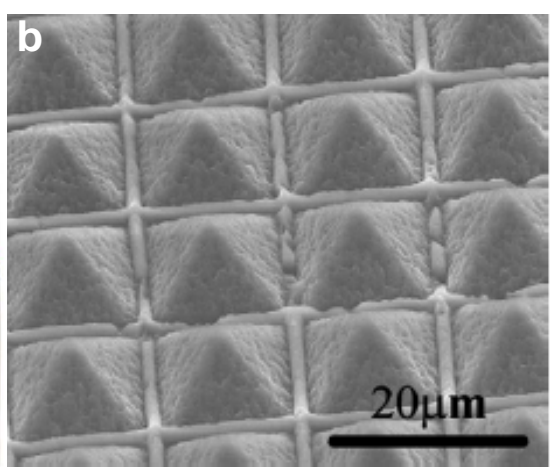

e

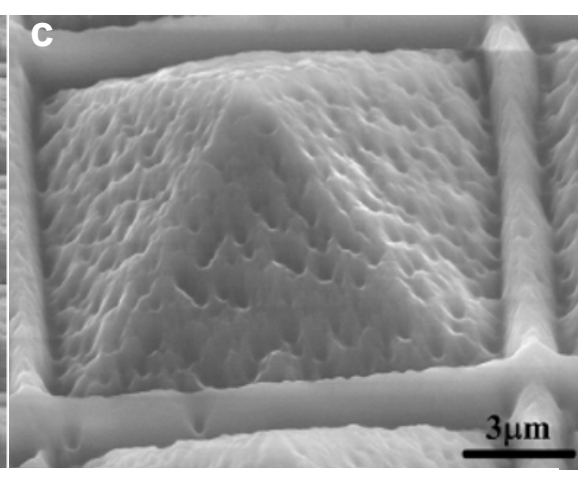

f
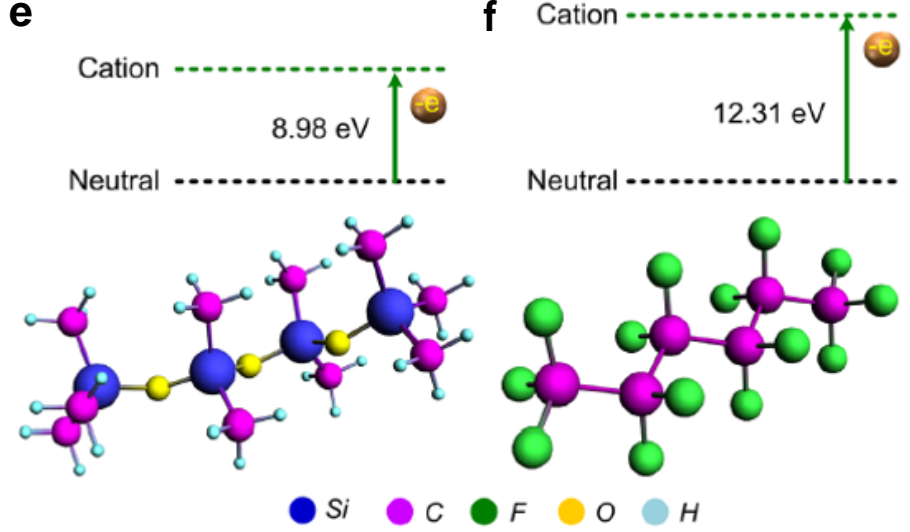

Figure 2. (a) Schematic of fluorocarbon plasma modification process. (b) SEM and (c) highmagnification SEM images of PDMS hierarchical structures after plasma treatment. (d) The variation of voltage output versus plasma treating cycles. (e,f)Calculated vertical ionization energy of model complexes of (e) pristine PDMS layer and (f) plasma deposited fluorocarbon layer. Higher ionization energy was obtained from the fluorocarbon treated film, meaning the promoted electron attracting capability of the modified PDMS. Reprinted with permission from ref 63. Copyright 2014, Elsevier. 

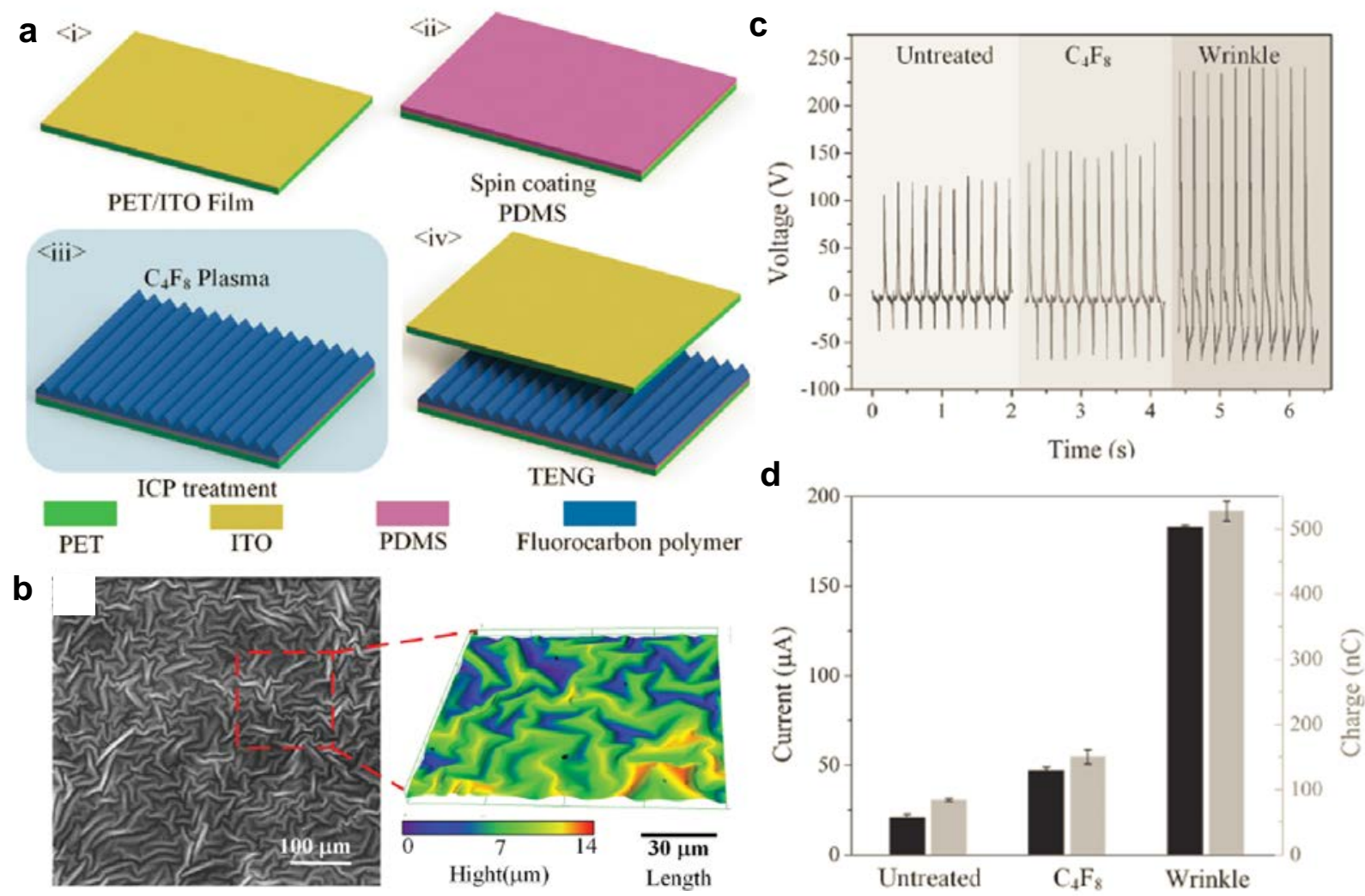

Figure 3. (a) Schematic of the wrinkled PDMS fabrication procedures, including spin coating of PDMS on PET/ITO substrate, $\mathrm{C}_{4} \mathrm{~F}_{8}$ plasma treating and TENG assembly. (b) SEM and laser scanning microscope (LSM) image of the wrinkled PDMS film. (c) Voltage output of TENGs made from untreated, $\mathrm{C}_{4} \mathrm{~F}_{8}$ plasma treated and wrinkled PDMS films. (d) The current and charge comparison of corresponding TENGs. Reprinted with permission from ref 64. Copyright 2015, Wiley-VCH. 

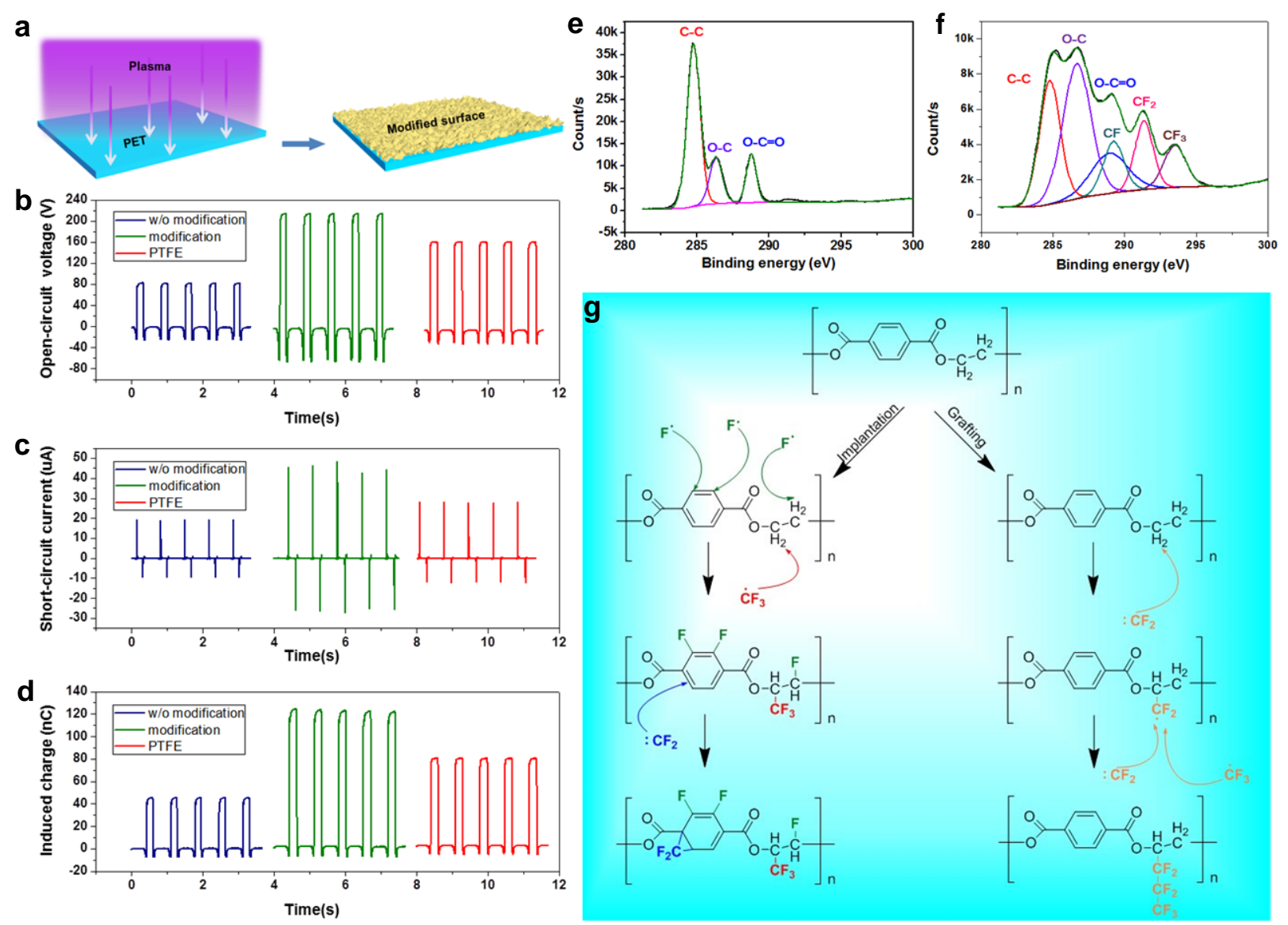

Figure 4. (a) Schematic illustration of ICP plasma modification process and the modified PET surface. (b) Open-circuit voltage, (c) short-circuit current, and (d) induced charge of TENGs assembled from pristine PET, plasma treated PET and PTFE films. (e,f) High-resolution XPS characterization and devolution of the C1s peak from the pristine (e) and modified (f) PET surfaces. (g) Schematics of two fluorination mechanisms during the plasma treatment. Reprinted with permission from ref 65. Copyright 2015, Wiley-VCH. 

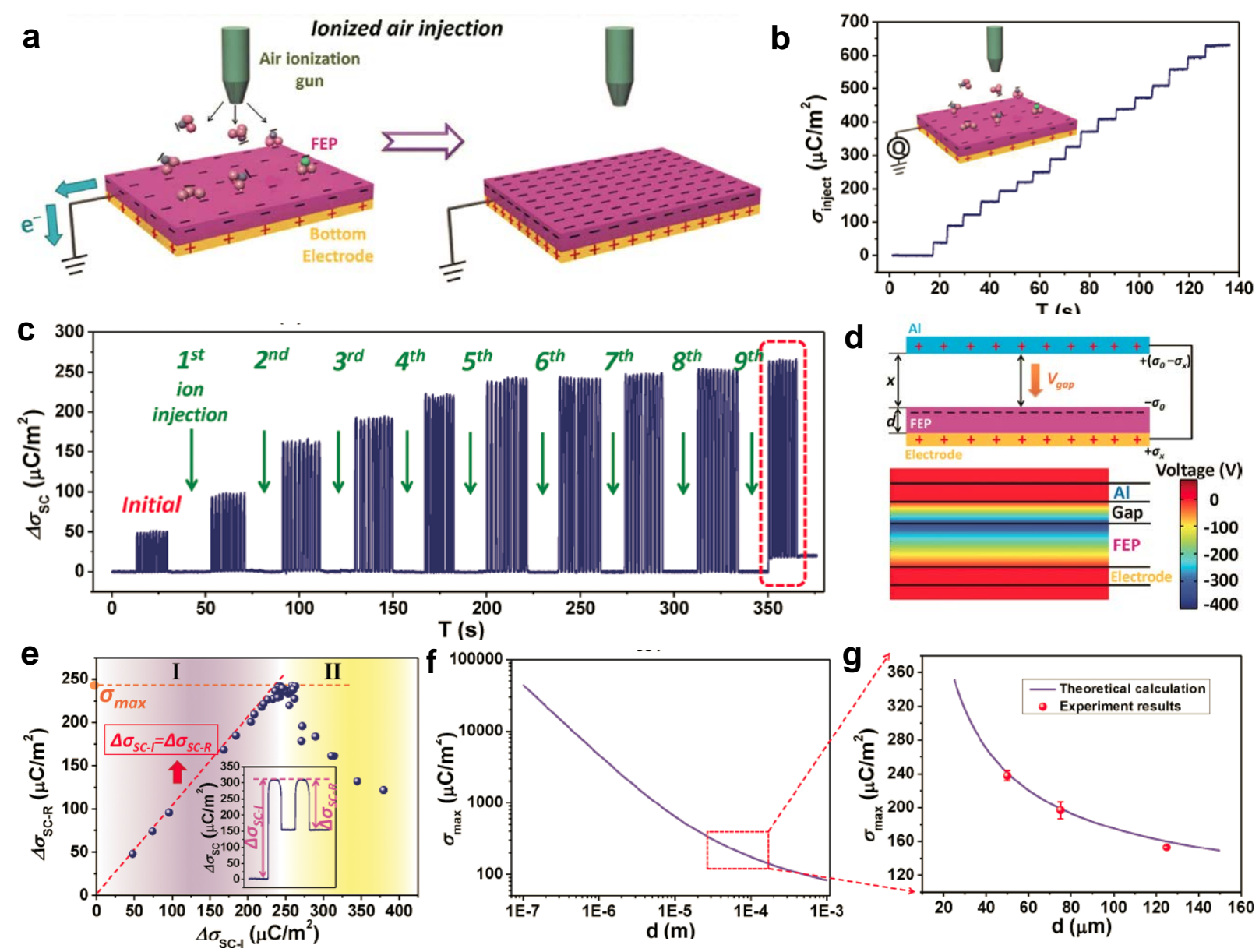

Figure 5. (a) Schematic illustration of injecting negative ions onto FEP surface with air ionization gun. The back electrode was grounded to maximizing the surface charge density. (b) In situ measurement of charge transfer from the ground to back electrode of FEP film during the step-by-step ion injections. (c) The variation of short-circuit charge density as a function of ion injection cycles. (d) Schematic and numerically calculated potential distribution across the $\mathrm{Al}$ and FEP gap, showing the presence of voltage drop in the gap, which could cause the breakdown of the air. (e) The relationship between the initial short-circuit charge density $\left(\Delta \sigma_{S C-I}\right)$ from the first pressing motion of TENG and the short-circuit charge density $\left(\Delta \sigma_{S C-R}\right)$ in the remaining cycles. Inset is the magnified curve for comparing $\Delta \sigma_{S C-I}$ and $\Delta \sigma_{S C-R .}$ (f) Theoretically calculated relationship between the maximum surface charge density $\left(\sigma_{\max }\right)$ and the film thickness $\mathrm{d}$ of FEP. (g) Plot of the above curve in the range of 20-150 $\mu \mathrm{m}$ region and three points of experimentally measured $\sigma_{\max }$ for the d of 50, 75 and $125 \mu \mathrm{m}$. Reprinted with permission from ref 70. Copyright 2014, Wiley-VCH. 

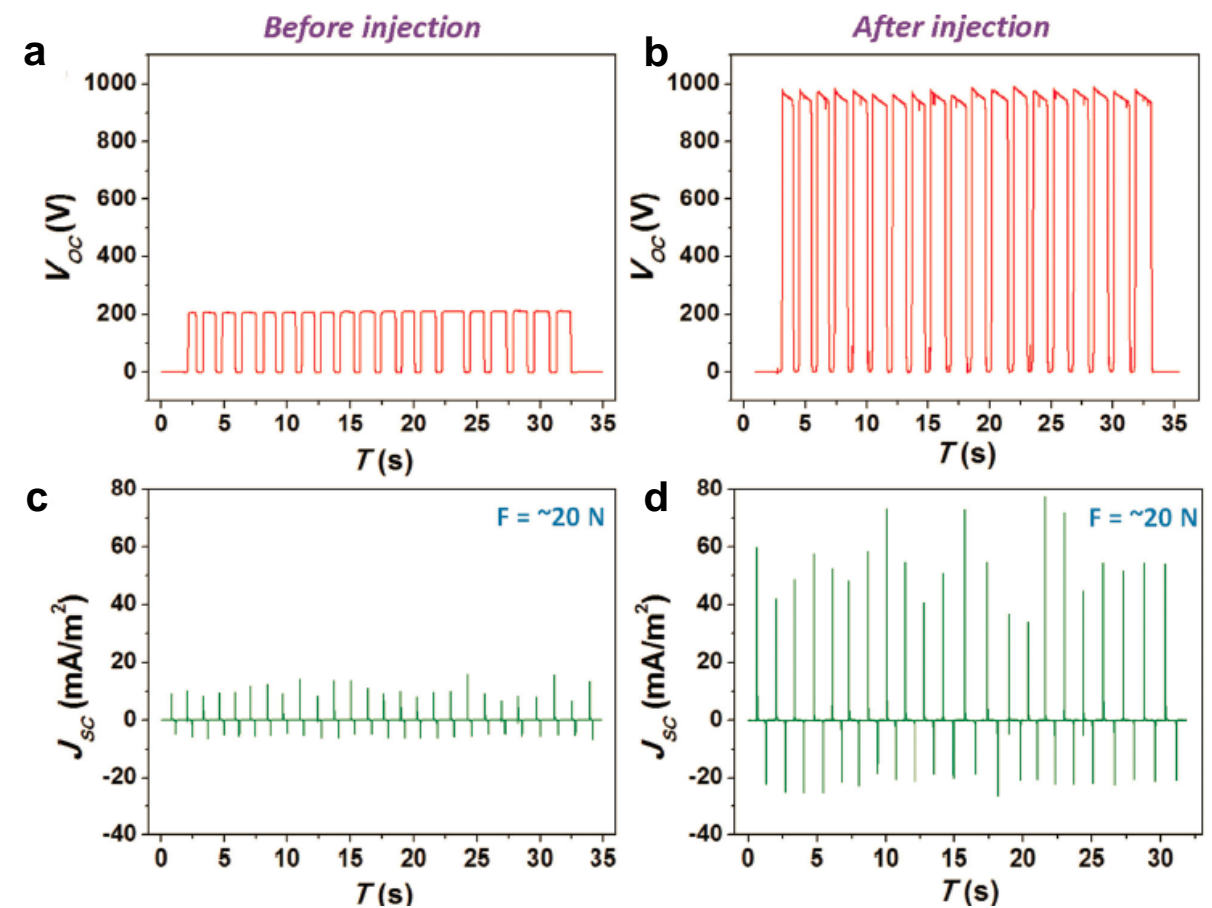

e
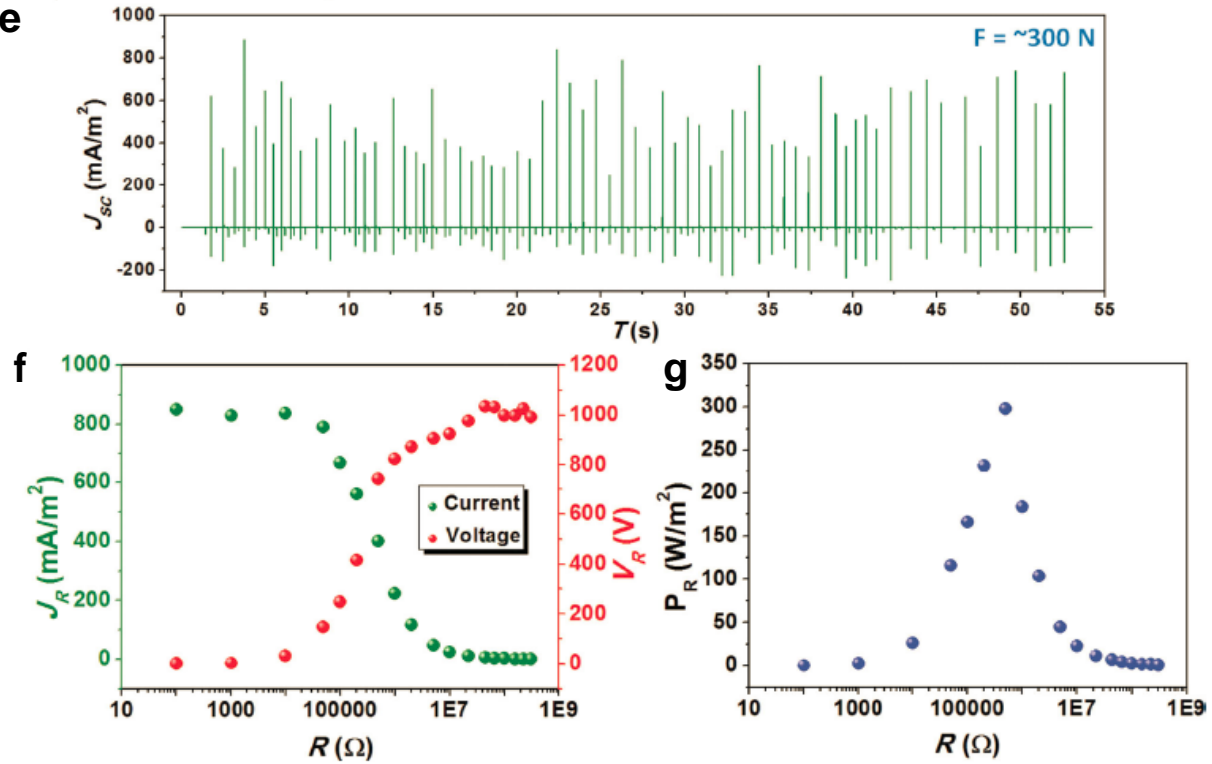

Figure 6. (a,b) $V_{o c}$ of TENG (a) before and (b) after the ion injection process. (c,d) $J_{s c}$ of TENG (c) before and (b) after the ion injection process with a pressing force of $\sim 20 \mathrm{~N}$. (e) $J_{s c}$ of TENG after ion injection with a deformation force of $\sim 300 \mathrm{~N}$. (f) The $V_{o c}$ and $J_{s c}$ variations of ion injected TENG versus different external load resistances. (g) Power density variation of ion injected TENG as a function of different external load resistances.Reprinted with permission from ref 70. Copyright 2014, Wiley-VCH. 

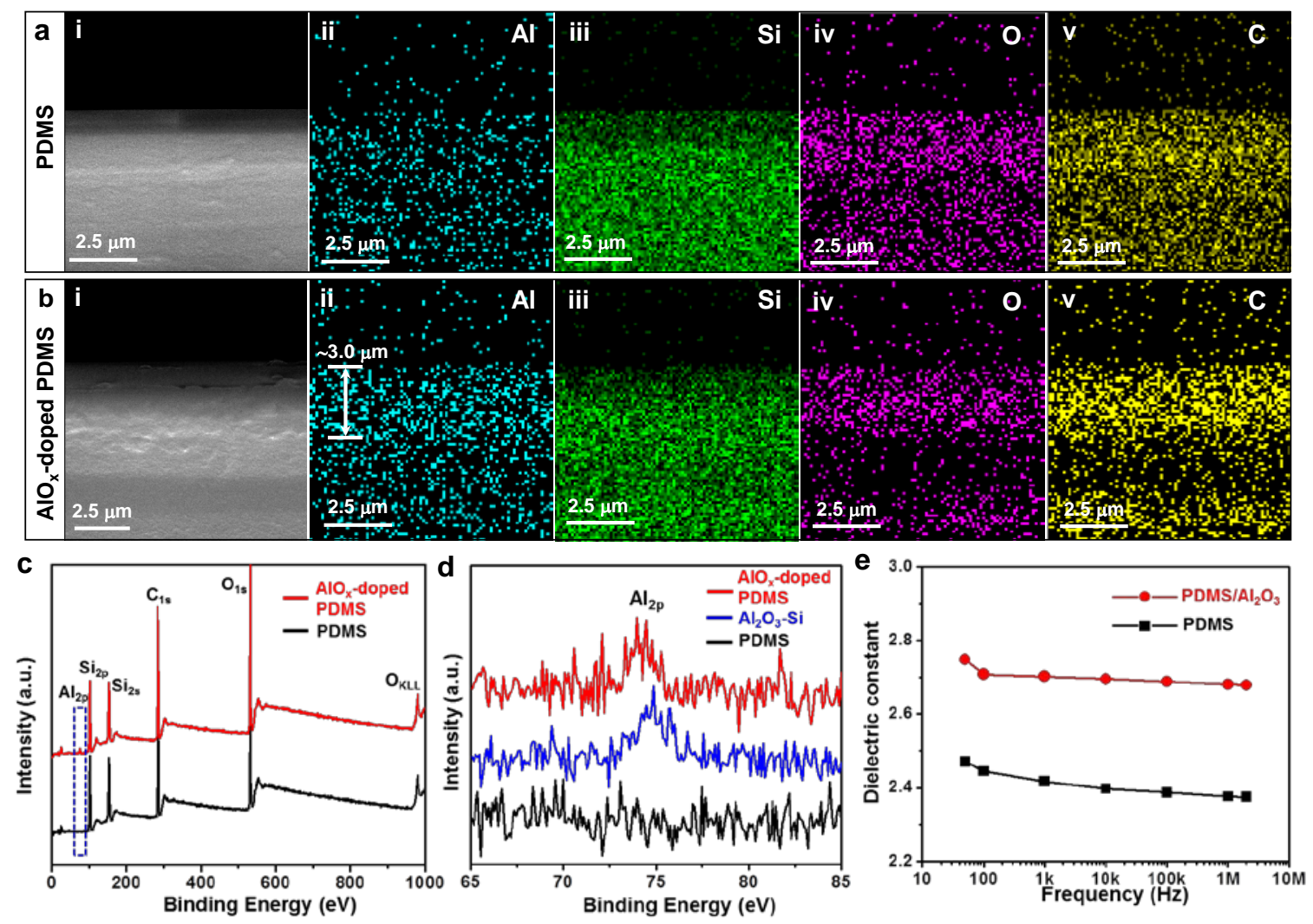

Figure 7. (a, b) Cross-sectional SEM images (i) and EDS mappings of a pristine PDMS film (a) and an $\mathrm{AlO}_{\mathrm{x}}$-doped PDMS film (b) for $\mathrm{Al}, \mathrm{Si}, \mathrm{O}$, and $\mathrm{C}$ elements (ii-v). (c) Full range XPS spectra acquired from pristine PDMS and $\mathrm{AlO}_{\mathrm{x}}$-doped PDMS. Small Al 2p peak was identified on the spectrum of $\mathrm{AlO}_{\mathrm{x}}$-doped PDMS. (d) Al 2p scan for pristine PDMS, $\mathrm{AlO}_{\mathrm{x}}$-doped PDMS film and $\mathrm{ALD}$ grown $\mathrm{Al}_{2} \mathrm{O}_{3}$ on silicon substrate. The peak position shift indicates the presence of defective oxides in $\mathrm{AlO}_{\mathrm{x}}$-doped PDMS film. (e) Dielectric constant measured from pristine PDMS and $\mathrm{AlO}_{\mathrm{x}}$-doped PDMS, showing increased dielectric constant of PDMS due to the $\mathrm{AlO}_{\mathrm{x}}$ doping. Reprinted with permission from ref 79. Copyright 2015, Wiley-VCH. 
$\mathbf{a}$
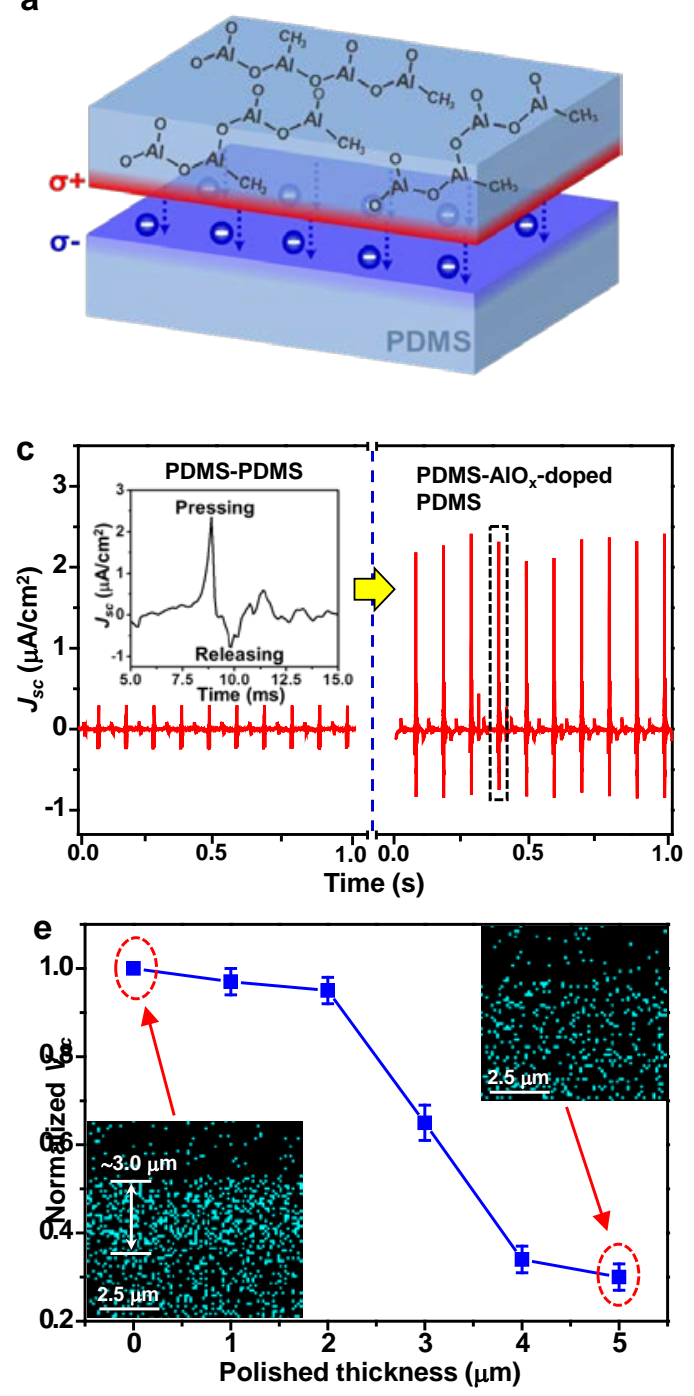
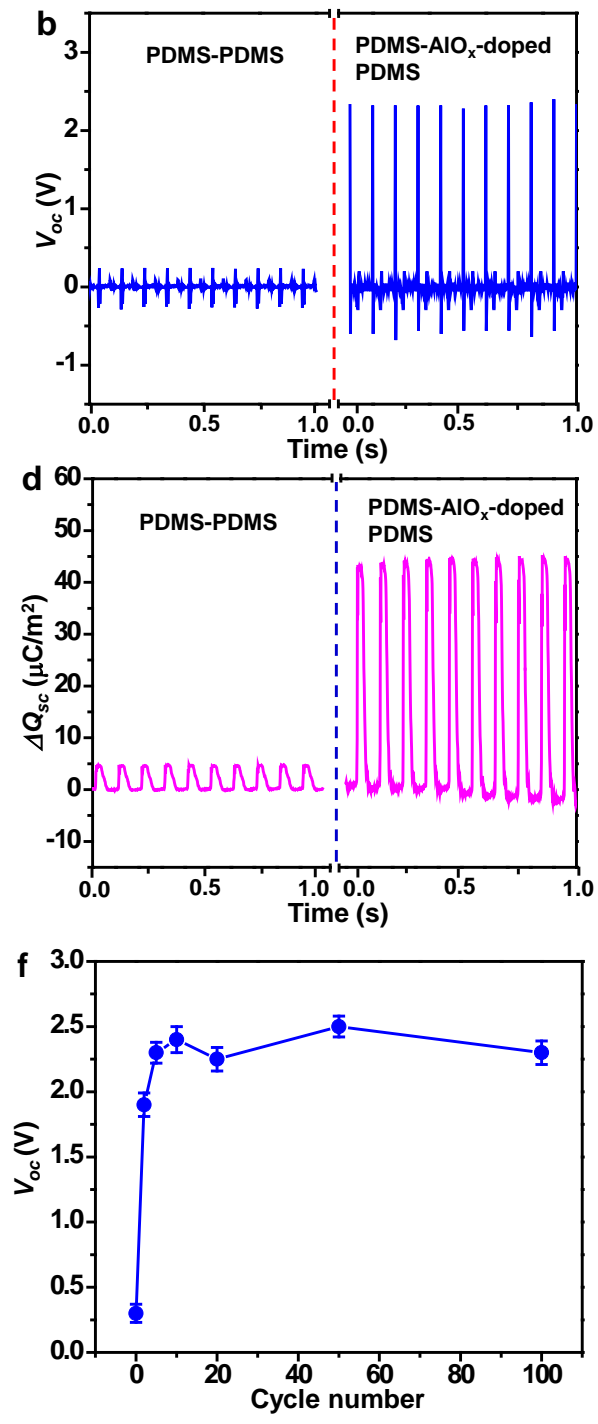

Figure 8. (a) Schematic of charge redistribution between pristine PDMS and $\mathrm{AlO}_{\mathrm{x}}$-doped PDMS films upon contact as a consequence of triboelectrification. (b) The $V_{o c}$, (c) $J_{s c}$ and (d) charge transfer amount under short-circuit condition $\left(\Delta Q_{s c}\right)$ of TENGs built from PDMS with and without $\mathrm{AlO}_{\mathrm{x}}$ doping. The inset of Figure 2c is the enlarged current output (dashed square box on Figure 2c) generated by one pressing and releasing cycle. (e) Normalized $V_{o c}$ of TENGs as a function of the material thickness that was polished off. Voltage output of $\mathrm{AlO}_{\mathrm{x}}$-doped PDMS film without any polishing was defined as unit. Insets are Al element EDS mapping of $\mathrm{AlO}_{\mathrm{x}^{-}}$ doped PDMS films before (left) and after (right) $\sim 5 \mu \mathrm{m}$ polishing. (f) $V_{o c}$ variation versus the doping cycles of $\mathrm{AlO}_{\mathrm{x}}$ on PDMS film.Reprinted with permission from ref 79. Copyright 2015, Wiley-VCH. 
a
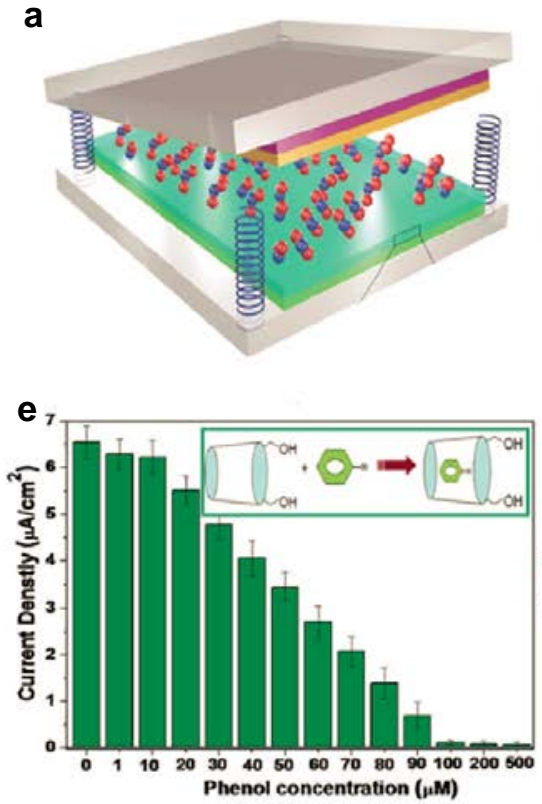

b

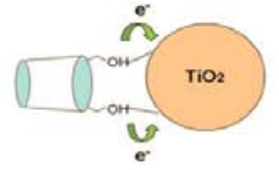

C
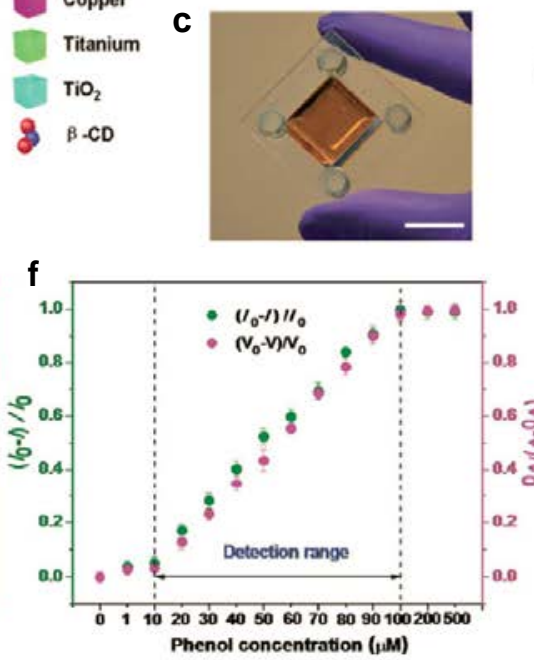
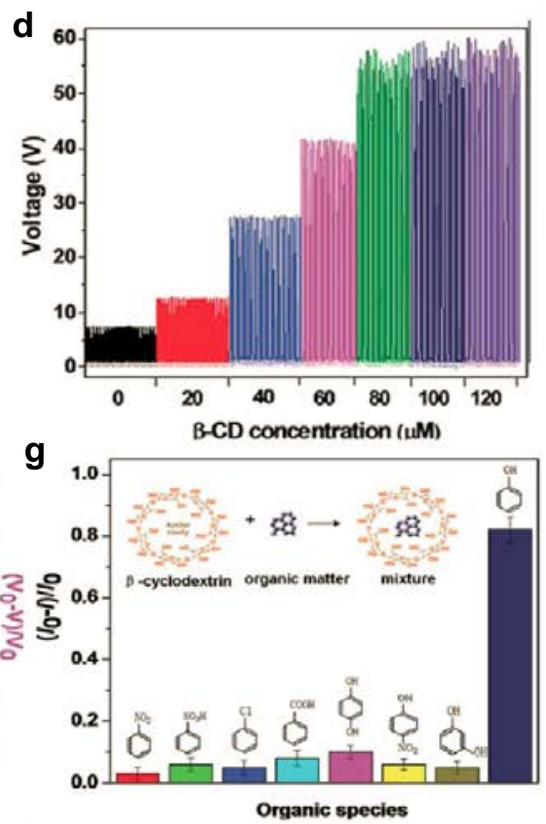

Figure 9. (a) Schematic of TENG device with $\beta$-cyclodextrin ( $\beta$-CD) modifed surface for phenol detction. (b) Schematic illustrations of electron transfer from hydroxyl group of $\beta-\mathrm{CD}$ to $\mathrm{TiO}_{2}$ nanowires. (c) A photograph of as-fabricated TENG device. The scale bar is $2 \mathrm{~cm}$. (d) Voltage output increment of $\beta$-CD modified TENGs with the increase of $\beta$-CD concenrations. (e) Current density reduction of phenol treated TENGs with the increase of phenol concentration. (f) The determination of sensitivity and detecting range of the TENG sensor in terms of both current and voltage output. (g) The current ratios of TENGs treated by various chemcials, showing particlular effective sensing function for phenol. Inset is the demonstration of reaction mechnism between $\beta$-CD and organic species.Reprinted with permission from ref 85. Copyright 2015, Royal Society of Chemistry. 
a
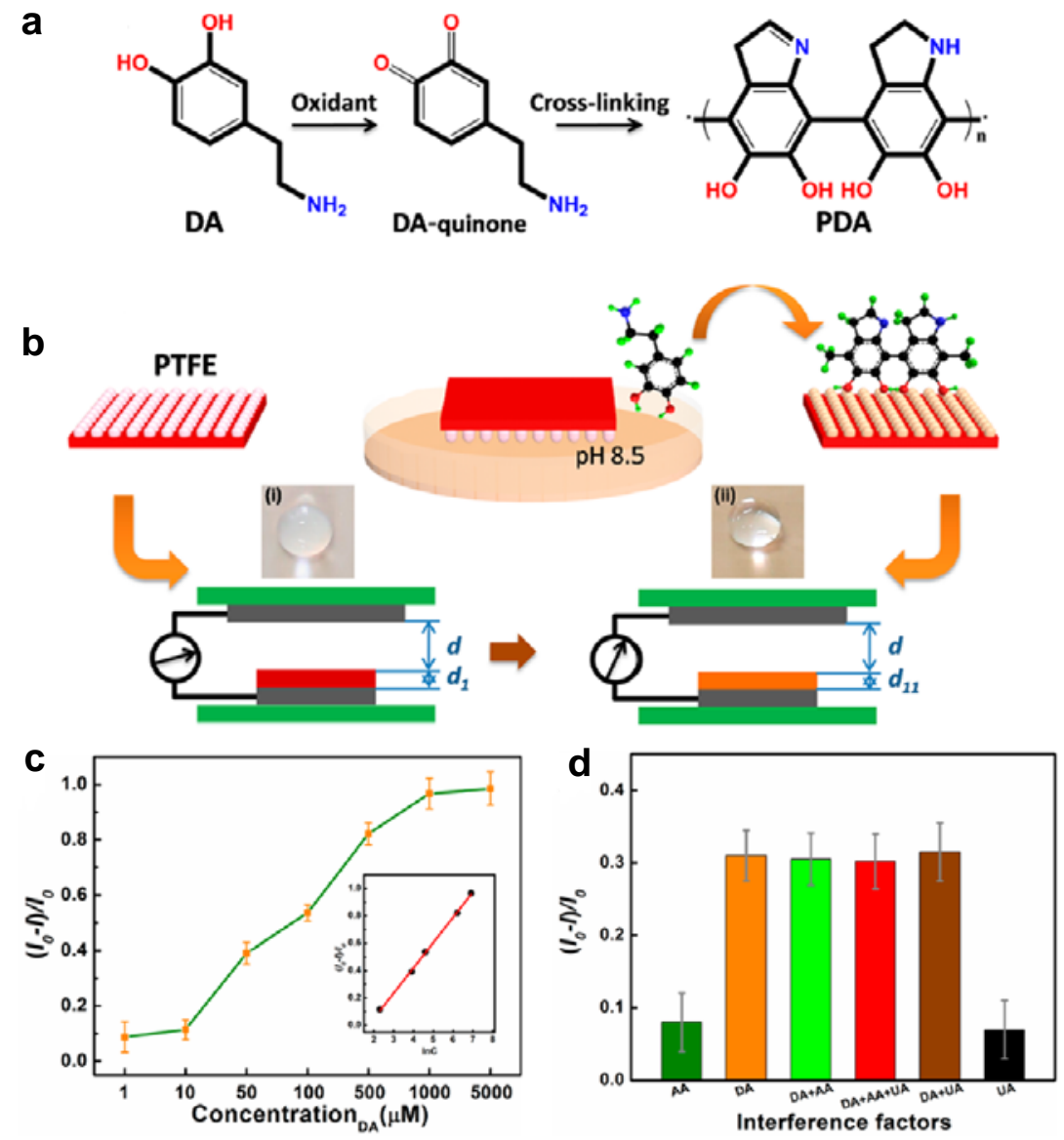

Figure 10. (a) Schematic of the reaction mechanism for dopmine (DA) polymerization. (b) Schematic illustration of polydopamine (PDA) depostion on PTFE surface and the inluence of PDA on the voltage output of TENG. Insets are photographs of water droplet on the surface of pristine (i) and PDA modified (ii) PTFE. (c) Sensitivity characterization of the TENG sensor for the DA detection. Inset is a plot showing the linear relationship between the current ratio and Napierian logarithm of the DA concentration. (d) Selectivity determination of the TENG sensor by introducing interference factors including ascobic acid (AA) and uric acid (UA). Large current ratio can only be obtained in the solution that contains DA, implying the good selectivity. Reprinted with permission from ref 88. Copyright 2015, American Chemical Society. 